Annals of Mathematics, 155 (2002), 235-280

\title{
Stability of blow-up profile and lower bounds for blow-up rate for the critical generalized $\mathrm{KdV}$ equation
}

\author{
By Yvan Martel and Frank Merle
}

\begin{abstract}
The generalized Korteweg-de Vries equations are a class of Hamiltonian systems in infinite dimension derived from the $\mathrm{KdV}$ equation where the quadratic term is replaced by a higher order power term. These equations have two conservation laws in the energy space $H^{1}$ ( $L^{2}$ norm and energy). We consider in this paper the critical generalized KdV equation, which corresponds to the smallest power of the nonlinearity such that the two conservation laws do not imply a bound in $H^{1}$ uniform in time for all $H^{1}$ solutions (and thus global existence).

From [15], there do exist for this equation solutions $u(t)$ such that $|u(t)|_{H^{1}} \rightarrow+\infty$ as $t \uparrow T$, where $T \leq+\infty$ (we call them blow-up solutions). The question is to describe, in a qualitative way, how blow up occurs.

For solutions with $L^{2}$ mass close to the minimal mass allowing blow up and with decay in $L^{2}$ at the right, we prove after rescaling and translation which leave invariant the $L^{2}$ norm that the solution converges to a universal profile locally in space at the blow-up time $T$. From the nature of this profile, we improve the standard lower bound on the blow-up rate for finite time blow-up solutions.
\end{abstract}

\section{Introduction}

1.1. We consider in this paper the critical generalized Korteweg-de Vries equation:

$$
\begin{cases}u_{t}+\left(u_{x x}+u^{5}\right)_{x}=0, & (t, x) \in \mathbf{R}^{+} \times \mathbf{R}, \\ u(0, x)=u_{0}(x), & x \in \mathbf{R},\end{cases}
$$

for $u_{0} \in H^{1}(\mathbf{R})$. It is a special case of the generalized Korteweg-de Vries equations, $p \geq 2$ integer:

$$
\begin{cases}u_{t}+\left(u_{x x}+u^{p}\right)_{x}=0, & (t, x) \in \mathbf{R}^{+} \times \mathbf{R}, \\ u(0, x)=u_{0}(x), & x \in \mathbf{R} .\end{cases}
$$


The case $p=2$ corresponds to the KdV equation (see Korteweg and de Vries $[9]$ ), and $p=3$ to the modified $\mathrm{KdV}$ equation. These two cases have been studied extensively for being completely integrable (see, for example, Lax [10] and Miura [16]). For all $p$, these equations are considered as universal models of Hamiltonian systems. From this Hamiltonian structure, there are two conservation laws

$$
\begin{aligned}
\int u^{2}(t) & =\int u_{0}^{2} \text { (mass conservation), } \\
\frac{1}{2} \int u_{x}^{2}(t)-\frac{1}{p+1} \int u^{p+1}(t) & =\frac{1}{2} \int u_{0 x}^{2}-\frac{1}{p+1} \int u_{0}^{p+1}
\end{aligned}
$$

(energy conservation).

From [8], we have the following existence and uniqueness result in the energy space $H^{1}(\mathbf{R})$ : for $u_{0} \in H^{1}(\mathbf{R})$, there exists $T>0$ and a unique maximal solution $u \in C\left([0, T), H^{1}(\mathbf{R})\right)$ of $(2)$ on $[0, T)$. Moreover, either $T=+\infty$, or $T<+\infty$, and then $|u(t)|_{H^{1}} \rightarrow+\infty$, as $t \uparrow T$. In addition, for all $t \in[0, T)$, (3) and (4) are satisfied. For equation (1), the local Cauchy problem is also well posed in $L^{2}(\mathbf{R})$ (see [8]). We refer to Kato [7] and Ginibre and Tsutsumi [6] for previous results on the well-posedness of the Cauchy problem for (2). See Bourgain [3] for the periodic case.

For $p<5$ (the subcritical case), as a consequence of the GagliardoNirenberg inequality, all solutions in $H^{1}$ are global and bounded in time.

In this paper, we consider only the critical case $p=5$. We define the energy

$$
E(u)=\frac{1}{2} \int u_{x}^{2}-\frac{1}{6} \int u^{6} .
$$

We consider solutions in the energy space $H^{1}(\mathbf{R})$. Let us introduce the ground state $Q$, unique positive solution (up to translation) of

$$
Q_{x x}+Q^{5}=Q, \quad Q \in H^{1}(\mathbf{R}), \quad \text { or equivalently } \quad Q(x)=\frac{3^{1 / 4}}{\operatorname{ch}^{1 / 2}(2 x)}
$$

Note that $u(t, x)=Q(x-t)$ is a special solution of $(1)$, and $E(Q)=0$.

On the one hand, the variational characterization of $Q$; i.e.: for $v \in$ $H^{1}(\mathbf{R})$,

$$
\begin{aligned}
& \text { if } 0<\int v^{2} \leq \int Q^{2} \text { and } E(v) \leq 0 \\
& \text { then there exists } \lambda_{0}>0, x_{0} \in \mathbf{R} / v=\lambda_{0}^{1 / 2} Q\left(\lambda_{0}\left(x-x_{0}\right)\right) \text {, }
\end{aligned}
$$

which provides the following Gagliardo-Nirenberg inequality with best constant 
(see Weinstein [17]):

$$
\text { for all } v \in H^{1}(\mathbf{R}), \quad \frac{1}{6} \int v^{6} \leq \frac{1}{2}\left(\frac{\int v^{2}}{\int Q^{2}}\right)^{2} \int v_{x}^{2},
$$

implies that for $\left|u_{0}\right|_{L^{2}}<|Q|_{L^{2}}$, the solution $u(t)$ is global and uniformly bounded in $H^{1}$.

On the other hand, for $\left|u_{0}\right|_{L^{2}}>|Q|_{L^{2}}$ there is no obstruction to blow up from energy-type arguments. Existence of solutions of (1) blowing up in finite or infinite time in the energy space $H^{1}$ has been proved by Merle (see [15] and also Martel and Merle [12]). More precisely:

There exists $\alpha_{0}>0$ such that for all $u_{0} \in H^{1}(\mathbf{R})$, if $E\left(u_{0}\right)<0$ and $\int u_{0}^{2}<\int Q^{2}+\alpha_{0}$, then the solution $u(t)$ blows up in $H^{1}$ in finite or infinite time.

Note that numerical observations suggest existence of blow up in finite time; see Bona et al. [1]. The argument for the blow-up proof in [15] is not direct. Arguing by contradiction, we consider a limit object, recurrent in time, as $t \rightarrow+\infty$. The idea is to show that the recurrence in time yields some rigidity on this object. Then, we are able to prove both elliptic and oscillatory integral type estimates on this limit solution; together with the three conservation laws (mass, energy and the additional invariant $\int u(t, x) d x$ when $u_{0} \in L^{1}$ ), this gives a contradiction with the Liouville Theorem in [12].

Moreover, the quantity $|Q|_{L^{2}}$ is the minimal amount of $L^{2}$ norm that concentrates at blow-up time, in the sense that for some function $x(t)$, we have for all $\varepsilon_{0}>0, \underline{\lim }_{t \uparrow T} \int_{|x-x(t)| \leq \varepsilon_{0}}|u(t)|^{2} \geq \int Q^{2}$.

In the study of the blow-up phenomenon, there are two main questions: the profile at blow-up time (in some smaller scale, describing the blow-up dynamics) and the rate of blow up of the solutions. These two questions are clearly linked. We consider the question of the blow-up profile for initial data as in the blow-up result, i.e. with $\int u_{0}^{2} \leq \int Q^{2}+\alpha_{0}$.

The first result of this paper is a characterization of the blow-up profile, which is $Q$, up to the invariances of the equation. This is, in some sense, a generalization of the Liouville theorem in [12], and of its corollary which says that any bounded solution, starting close to $Q$ in $H^{1}$, converges locally in space to $Q$ for large time.

TheOREM 1 (Stability of $Q$ as a blow-up profile). There exists $\alpha_{0}>0$ such that if $u_{0} \in H^{1}(\mathbf{R})$ satisfies

$$
\int u_{0}^{2}<\int Q^{2}+\alpha_{0}
$$

and if the solution $u(t)$ of (1) blows up in finite or infinite time $T>0$, then for all $0 \leq t<T$, there exists $\lambda(t)>0$ and $x(t) \in \mathbf{R}$ such that either 


$$
\lambda^{1 / 2}(t) u(t, \lambda(t) x+x(t)) \rightarrow Q \quad \text { as } t \uparrow T \text { in } H^{1}(\mathbf{R}) \text { weak },
$$

or

$$
-\lambda^{1 / 2}(t) u(t, \lambda(t) x+x(t)) \rightarrow Q \quad \text { as } t \uparrow T \text { in } H^{1}(\mathbf{R}) \text { weak. }
$$

Remark. Note that the alternative in Theorem 1 comes from the fact that if $u(t, x)$ is a solution of (1) then $-u(t, x)$ is also a solution of (1).

There is no such result of determination of blow-up profile in Hamiltonian systems or more generally in evolution partial differential equations except for diffusion equations where the existence of Liapunov functions plays a fundamental role. Indeed, in the case of the nonlinear heat equations $u_{t}=\Delta u+u^{p}$ in $\mathbf{R}^{N}$, with some restriction on $p$, the blow-up rate and profile (and their stability) have been determined; see for example Giga and Kohn [5] and FermanianKammerer, Merle and Zaag [4].

Remark. If $E\left(u_{0}\right)<0$ in Theorem 1 , we actually know that $u(t)$ blows up in finite or infinite time; see [15]. Note that the result implies the stability and the universality of the blow-up profile in the region $\int u_{0}^{2} \leq \int Q^{2}+\alpha_{0}$ and $E\left(u_{0}\right)<0$.

We consider now an application of this result to obtain a refined lower bound on the blow-up rate. In particular we exclude some candidates (deduced from a scaling argument) of the blow-up rate. By a scaling argument and the resolution of the Cauchy problem, if $u(t)$ is a solution blowing up at some finite time $T>0$, then for some $C>0$,

$$
\text { for all } t_{0} \in[0, T), \quad\left|u_{x}\left(t_{0}\right)\right|_{L^{2}} \geq \frac{C}{\left(T-t_{0}\right)^{1 / 3}} .
$$

Indeed, consider

$$
v_{t_{0}}(t, x)=\left|u_{x}\left(t_{0}\right)\right|_{L^{2}}^{-1 / 2} u\left(t_{0}+\left|u_{x}\left(t_{0}\right)\right|_{L^{2}}^{-3} t,\left|u_{x}\left(t_{0}\right)\right|_{L^{2}}^{-1} x\right) ;
$$

$v_{t_{0}}$ is a solution of (1) by scaling invariance. We have $\left|v_{t_{0} x}\right|_{L^{2}}+\left|v_{t_{0}}\right|_{L^{2}} \leq C$, and so by the resolution of the Cauchy problem locally in time by a fixed-point argument (see [8]), there exists $\tau>0$, independent of $t_{0}$, such that $v_{t_{0}}(t)$ is defined on $[0, \tau]$. Therefore, $t_{0}+\left|u_{x}\left(t_{0}\right)\right|_{L^{2}}^{-3} \tau<T$, which is the desired result. Theorem 1 implies that this lower bound represents the exact blow-up rate for no solution with small $L^{2}$ mass. Indeed, we have the following theorem.

TheOREM 2 (Lower bound on the blow-up rate). There exists $\alpha_{0}>0$ such that if $u_{0} \in H^{1}(\mathbf{R})$ satisfies

$$
\int u_{0}^{2}<\int Q^{2}+\alpha_{0}
$$

and if the solution $u(t)$ of (1) blows up in finite time $T>0$, then

$$
\lim _{t \uparrow T}(T-t)^{1 / 3}\left|u_{x}(t)\right|_{L^{2}}=+\infty .
$$


Remark. For the critical KdV equation, Bona and Weissler [2] constructed explicit solutions of (1) with self similar blow up. However these solutions are not in the physical space $L^{2}$ and also exist in the linear context. In fact, Theorem 2 excludes this type of blow-up rate for solutions with $L^{2}$ mass close to the minimal mass allowing blow up, and we expect this result to extend to all initial data in $H^{1}$.

This phenomenon, which forces the solutions to blow up more quickly than the self similar rate in the energy space, seems to be typical of Hamiltonian systems in PDE with infinite speed of propagation. It is still an open problem for the critical nonlinear Schrödinger equation (NLSE):

$$
\left\{\begin{array}{l}
\mathrm{i} u_{t}=-u_{x x}-|u|^{4} u, \quad(t, x) \in \mathbf{R}^{+} \times \mathbf{R}, \\
u(0, x)=u_{0}(x), \quad x \in \mathbf{R} .
\end{array}\right.
$$

Indeed, the rate predicted by scaling arguments in energy space, which is $\left|u_{x}(t)\right|_{L^{2}} \sim C / \sqrt{T-t}$, should be not relevant for blow-up solution in $H^{1}$. Evidence for this fact is on the one hand the existence of explicit solutions of the nonlinear critical Schrödinger equation with a blow-up rate $C /(T-t)$, and on the other hand numerical results suggesting different blow-up rates. See for example Merle [14] for more information about blow up for NLSE. Note that for the Zakharov system (critical NLSE coupled with a wave equation), the optimal lower bound for the blow-up rate $(C /(T-t))$ has been derived by Merle [13].

The second author thanks Stanford University where part of this work was done.

1.2. Strategy of the proof. First, as in [12] and [15], from the fact that $\int u_{0}^{2}<\int Q^{2}+\alpha_{0}$, with $\alpha_{0}$ small, a parametrization of the problem allows us to see the evolution in time of the size and location of the solution. Note that for all $\lambda_{0}>0, x_{0} \in \mathbf{R}, v_{\lambda_{0}, x_{0}}(x)=\lambda_{0}^{1 / 2} Q\left(\lambda_{0}\left(x-x_{0}\right)\right)$ is such that $\int v_{\lambda_{0}, x_{0}}^{2}=\int Q^{2}$ and $E\left(v_{\lambda_{0}, x_{0}}\right)=0$. Therefore, there is no obstruction from the conservation laws to the existence of a solution of the form

$$
u(t, x) \sim \lambda^{-1 / 2}(t) Q\left(\lambda^{-1}(t)(x-x(t))\right) .
$$

In fact, it follows from variational arguments that a blow-up solution with $\alpha_{0}$ small is close in $H^{1}$ to the set $\left\{v_{\lambda_{0}, x_{0}}\right\}$, for all time close to the blow-up time. Indeed, set

$$
\varepsilon(t, y)=\lambda^{1 / 2}(t) u(t, \lambda(t) y+x(t))-Q(y),
$$

for $\lambda(t)>0, x(t)$, two $C^{1}$ functions to be chosen later. For a suitable choice of $\lambda(t)$ and $x(t), \varepsilon(t)$ is uniformly small in time in $H^{1}(\mathbf{R})$. Change the time variable as follows:

$$
s=\int_{0}^{t} \frac{d t^{\prime}}{\lambda^{3}\left(t^{\prime}\right)}, \quad \text { or equivalently, } \quad \frac{d s}{d t}=\frac{1}{\lambda^{3}}
$$


then $\varepsilon(s)$ satisfies, for $s \geq 0, y \in \mathbf{R}$,

$$
\begin{aligned}
\varepsilon_{s}= & (L \varepsilon)_{y}+\frac{\lambda_{s}}{\lambda}\left(\frac{Q}{2}+y Q_{y}\right)+\left(\frac{x_{s}}{\lambda}-1\right) Q_{y} \\
& +\frac{\lambda_{s}}{\lambda}\left(\frac{\varepsilon}{2}+y \varepsilon_{y}\right)+\left(\frac{x_{s}}{\lambda}-1\right) \varepsilon_{y}-\left(10 Q^{3} \varepsilon^{2}+10 Q^{2} \varepsilon^{3}+5 Q \varepsilon^{4}+\varepsilon^{5}\right)_{y}
\end{aligned}
$$

where

$$
L \varepsilon=-\varepsilon_{x x}+\varepsilon-5 Q^{4} \varepsilon=-\varepsilon_{x x}+\varepsilon-\frac{15}{\operatorname{ch}^{2}(2 x)} \varepsilon .
$$

(See Lemma 1 in [11].) Note that $x(t)$ and $\lambda(t)$ are geometrical parameters related to the two invariances of equation (1): respectively, translation and dilation invariances.

If, for all $t \geq 0, u(t)$ is sufficiently close to $Q$ in $H^{1}$, up to scaling and translation, we can define a unique $C^{1}$ function $s \rightarrow(\lambda(s), x(s))$ such that for all $s \geq 0$,

$$
\int y\left(\frac{Q}{2}+y Q_{y}\right) \varepsilon(s)=\int y Q_{y} \varepsilon(s)=0 .
$$

The reason to consider such orthogonality conditions on $\varepsilon(s)$ is the fact that they are adapted to a Virial-type identity on $\varepsilon(s)\left(\frac{d}{d s} \int y \varepsilon^{2}(s)\right.$; see $\left.\S 2.2\right)$. Indeed, these relations cancel some interactions in the Virial relation, and are one of the crucial tools of this paper.

Theorem 2 follows directly from Theorem 1 , and the fact that $u(t, x)=$ $Q(x-t)$ is a solution of (1) such that for all $t,\left|u_{x}(t)\right|_{L^{2}}=\left|Q_{x}\right|_{L^{2}}$. (See §4.1.) The proof of Theorem 1 is by contradiction. Assume that we have a solution for $\alpha_{0}$ small such that

$$
\varepsilon(s) \not 0 \text { in } H^{1}(\mathbf{R}) \text {, as } s \rightarrow+\infty .
$$

As in [15], the idea is to define a recurrent object as $t \rightarrow T$. From the property of recurrence of this object, and some almost monotonic in-time functional, this object has more properties (decay properties as $y \rightarrow-\infty$ ). The proof of nonexistence of such an object then concludes the proof of Theorem 1.

More precisely, define $s_{n} \rightarrow+\infty$ such that $\varepsilon\left(s_{n}\right) \rightarrow \widetilde{\varepsilon}(0) \not \equiv 0$, and $\widetilde{u}$ solution of (1) with $\widetilde{u}(0)=Q+\widetilde{\varepsilon}(0)$. This limit solution $\widetilde{u}(t)$ is associated to $\widetilde{\varepsilon}, \widetilde{\lambda}, \widetilde{x}$, and is such that $\widetilde{\lambda}(0)=1$. Define $\tau>0$ such that for all $s \in[0, \tau)$, $\frac{1}{1.1} \leq \tilde{\lambda}(t) \leq 1$. The contradiction follows from three facts.

(i) We first prove exponential decay on the left for $\widetilde{\varepsilon}$, in the sense that

$$
\text { for all } s \in[0, \tau) \text {, for all } y<0, \quad \widetilde{\varepsilon}(s, y) \mid \leq C\left(\alpha_{0}\right) e^{-\frac{|y|}{12}},
$$

where $C\left(\alpha_{0}\right) \rightarrow 0$ as $\alpha_{0} \rightarrow 0$. (See $\S 2.3$ and $\S 4.2$.)

We then conclude by using two dispersion relations giving information on the dynamics of $\widetilde{\varepsilon}(s)$ : 
(ii) an $L^{1}$ relation, involving a quantity of the type $\int \widetilde{\varepsilon}(s) V$, where $V$ is bounded such that $V(y) \rightarrow 1$ as $y \rightarrow-\infty$, and $V(y) \rightarrow 0$ as $y \rightarrow+\infty$.

(iii) an $L^{2}$ relation, which is a local Virial-type identity, i.e. an expression for $\frac{d}{d s} \int \Psi_{A} \widetilde{\varepsilon}^{2}$, where $\Psi_{A}$ is bounded and such that $\Psi_{A}(y) \sim y$ for $|y|<A$.

These three facts provide a contradiction on $\widetilde{\varepsilon}$ and $\widetilde{u}$, in the two possible regimes $\tau<+\infty$ or $\tau=+\infty$. (See $\S 3.2$ and $\S 4.2$.)

The paper is organized as follows. In Section 2, we establish fundamental relations. In Section 3, we study a simpler and more geometric case, where the limit object is more natural that in Theorem 1. This allows us to present the main ideas without the technical difficulties of Section 4 . We prove directly that for $\alpha_{0}$ small, the upper bound

$$
\left|u_{x}(t)\right|_{L^{2}} \leq \frac{C}{(T-t)^{1 / 3}}
$$

is not possible for blow-up solutions. Section 4 is then devoted to the proof of Theorems 1 and 2 .

\section{Energy and dispersive relations}

2.1. Decomposition of the solution. For $v \in H^{1}(\mathbf{R})$, let

$$
\alpha(v)=\int v^{2}-\int Q^{2}, \quad E(v)=\frac{1}{2} \int v_{x}^{2}-\frac{1}{6} \int v^{6} .
$$

For $\alpha_{0}>0$ to be fixed later, let $u_{0} \in H^{1}(\mathbf{R})$ be such that $\alpha\left(u_{0}\right) \leq \alpha_{0}$. Assume that the solution $u(t, x)$ of (1) blows up in finite or infinite time $T>0$ (which implies by $(6)$ that $\alpha\left(u_{0}\right) \geq 0$ ). Note that $-u(t, x)$ is also a solution of (1) with initial data $-u_{0}$, and with the same properties as $u(t, x)$.

First, we have the following decomposition of the solution, using variational tools, and elementary geometrical properties. This decomposition allows us to extract some finite dimensional approximation of the solution which gives in some sense the time evolution of its size and its location.

LEMma 1 (Sharp decomposition and modulation of the solution). There exists $\alpha_{1}>0$ such that if $\alpha_{0}<\alpha_{1}$ then there exist $0 \leq t\left(u_{0}\right)<T$ and continuous functions $\lambda:\left[t\left(u_{0}\right), T\right) \rightarrow(0,+\infty), x:\left[t\left(u_{0}\right), T\right) \rightarrow \mathbf{R}$, such that, for $v \equiv u$ or $v \equiv-u$, for all $t \in\left[t\left(u_{0}\right), T\right)$,

$$
\varepsilon(t, y)=\lambda^{1 / 2}(t) v(t, \lambda(t) y+x(t))-Q(y)
$$

satisfies the following properties: for all $t \in\left[t\left(u_{0}\right), T\right)$,

$$
\begin{gathered}
\int y Q_{y} \varepsilon(t)=\int y\left(\frac{Q}{2}+y Q_{y}\right) \varepsilon(t)=0, \\
\left|1-\lambda(t) \frac{\left|u_{x}(t)\right|_{L^{2}}}{\left|Q_{x}\right|_{L^{2}}}\right|+|\varepsilon(t)|_{H^{1}} \leq \delta^{\prime}\left(\alpha_{0}\right), \quad \text { where } \delta^{\prime}(\alpha) \rightarrow 0 \text { as } \alpha \rightarrow 0 .
\end{gathered}
$$


Remark. In the rest of this paper, we assume that $v=u$ in Lemma 1, by possibly replacing $u(t, x)$ by $-u(t, x)$.

Proof. The proof is similar to the one of Lemmas 1 and 2 in [15].

Step 1. We first prove that any blow-up solution with $L^{2}$ norm close to the $L^{2}$ norm of $Q$ is close to the set $\left\{ \pm \lambda_{0}^{-1 / 2} Q\left(\lambda_{0}^{-1}\left(x-x_{0}\right)\right) \mid \lambda_{0}>0, x_{0} \in \mathbf{R}\right\}$ for $t$ close enough to the blow-up time.

Claim. There exists $\alpha_{2}>0$ such that the following property holds true. For all $0<\alpha^{\prime} \leq \alpha_{2}$, there exists $\delta=\delta\left(\alpha^{\prime}\right)>0$, with $\delta\left(\alpha^{\prime}\right) \rightarrow 0$ as $\alpha^{\prime} \rightarrow 0$, such that for all $u \in H^{1}(\mathbf{R}), u \neq \equiv$, if

$$
\alpha(u) \leq \alpha^{\prime}, \quad E(u) \leq \alpha^{\prime} \int u_{x}^{2}
$$

then there exist $x_{0} \in \mathbf{R}$ and $\epsilon_{0} \in\{-1,1\}$ such that

$$
\left|Q-\epsilon_{0} \lambda_{0}^{1 / 2} u\left(\lambda_{0} x+x_{0}\right)\right|_{H^{1}} \leq \delta\left(\alpha^{\prime}\right)
$$

with $\lambda_{0}=\left|Q_{x}\right|_{L^{2}} /\left|u_{x}\right|_{L^{2}}$.

Proof of the claim. It follows from variational arguments. For the sake of contradiction, consider a sequence $\left(u_{n}\right)$ of functions in $H^{1}(\mathbf{R}), u_{n} \not \equiv 0$, such that

$$
\lim _{n \rightarrow \infty} \int u_{n}^{2} \leq \int Q^{2}, \quad \lim _{n \rightarrow \infty} \frac{E\left(u_{n}\right)}{\int u_{n x}^{2}} \leq 0 .
$$

Let us recall the following variational result, following the variational characterization of $Q$ (see Lemma 1 in [15]): if a sequence $\left(v_{n}\right)$ of $H^{1}$ functions satisfies:

$$
\lim _{n \rightarrow+\infty} \int v_{n}^{2}=\int Q^{2}, \quad \int v_{n x}^{2}=\int Q_{x}^{2}, \quad \varlimsup_{n \rightarrow+\infty} E\left(v_{n}\right) \leq 0,
$$

then there exists a sequence $\left(x_{n}\right)$ of $\mathbf{R}$, and $\epsilon_{0} \in\{-1,1\}$ such that

$$
\lim _{n \rightarrow \infty} \epsilon_{0} v_{n}\left(.+x_{n}\right)=Q \text { in } H^{1}(\mathbf{R}) \text { as } n \rightarrow \infty .
$$

We set

$$
\lambda_{n}=\frac{\left|Q_{x}\right|_{L^{2}}}{\left|u_{n x}\right|_{L^{2}}} \quad \text { and } \quad v_{n}=\lambda_{n}^{1 / 2} u_{n}\left(\lambda_{n} x\right) .
$$

Note that $\int v_{n}^{2}=\int u_{n}^{2}$ and $\int\left(v_{n}\right)_{x}^{2}=\int Q_{x}^{2}$. We prove that the sequence $v_{n}$ satisfies (15), which finishes the proof of the claim. 
Indeed, by Gagliardo-Nirenberg inequality with best constant (6), we have

$$
\frac{E\left(u_{n}\right)}{\int u_{n x}^{2}} \geq \frac{1}{2}\left(1-\frac{\int u_{n}^{2}}{\int Q^{2}}\right),
$$

and since $\lim _{n \rightarrow+\infty} \alpha\left(u_{n}\right) \leq 0, \lim _{n \rightarrow+\infty} \frac{E\left(u_{n}\right)}{\int u_{n x}^{2}} \leq 0$, it follows that

$$
\lim _{n \rightarrow \infty} \int u_{n}^{2}=\lim _{n \rightarrow \infty} \int v_{n}^{2}=\int Q^{2} \text { and } \lim _{n \rightarrow \infty} \frac{E\left(u_{n}\right)}{\int u_{n x}^{2}}=0 .
$$

By direct calculations, we have:

$$
E\left(v_{n}\right)=\frac{E\left(u_{n}\right)}{\int u_{n x}^{2}} \int Q_{x}^{2}, \quad \int\left(v_{n}\right)_{x}^{2}=\int Q_{x}^{2} .
$$

Therefore, the sequence $\left(v_{n}\right)$ satisfies (15).

Step 2. Modulation of the solution. By conservation of energy, we have for all $t \in[0, T), E(u(t))=E\left(u_{0}\right)$. Since $\lim _{t \uparrow T}\left|u_{x}(t)\right|_{L^{2}}=+\infty$, there exists $t_{0} \in[0, T)$ such that

$$
\text { for all } t \in\left[t_{0}, T\left[, \quad \alpha_{0} \int u_{x}^{2}(t) \geq E(u(t))=E\left(u_{0}\right) .\right.\right.
$$

Therefore, by step 1 , for all $t \in\left[t_{0}, T\right)$, there exist $x_{0}(t) \in \mathbf{R}$, and $\epsilon_{0}(t) \in$ $\{-1,1\}$ such that, with $\lambda_{0}(t)=\left|Q_{x}\right|_{L^{2}} /\left|u_{x}(t)\right|_{L^{2}}$,

$$
\left|Q-\epsilon_{0}(t) \lambda_{0}^{1 / 2}(t) u\left(t, \lambda_{0}(t) x+x_{0}(t)\right)\right|_{H^{1}} \leq \delta\left(\alpha_{0}\right) .
$$

As in Merle [15], if $\delta\left(\alpha_{0}\right)<|Q|_{L^{2}} / 4$ (which is true when $\alpha_{0}$ is small), then $\epsilon_{0}(t)$ is independent of $t$. Moreover, we can assume, with no restriction, that $\epsilon_{0}(t)=1$ (note that $-u(t, x)$ is also a solution of $(1)$ ).

Now, as in Lemma 2 of [15], we sharpen the decomposition; i.e. we choose $\lambda(t), x(t)$ close to $\lambda_{0}(t), x_{0}(t)$ such that $\varepsilon(t)=\lambda^{1 / 2}(t) u(t, \lambda(t) y+x(t))-Q(y)$ is small in $H^{1}$ and also satisfies suitable orthogonality conditions: for all $t \in$ $\left[t\left(u_{0}\right), T\right)$,

$$
\int y Q_{y} \varepsilon(t)=\int y\left(\frac{Q}{2}+y Q_{y}\right) \varepsilon(t)=0
$$

Here, we use the implicit function theorem. Note that with respect to Lemma 2 in [15], we have modified the orthogonality conditions. See also Part B of [12]. In the present case, we have

$$
\begin{aligned}
&\left(\frac{d}{d \lambda_{0}} \lambda_{0}^{1 / 2} Q\left(\lambda_{0} x+x_{0}\right)\right)_{\left(\lambda_{0}=1, x_{0}=0\right)} \\
&=\frac{Q}{2}+y Q_{y},\left(\frac{d}{d x_{0}} \lambda_{0}^{1 / 2} Q\left(\lambda_{0} x+x_{0}\right)\right)_{\left(\lambda_{0}=1, x_{0}=0\right)}=Q_{y},
\end{aligned}
$$


and the nondegeneracy conditions are satisfied since

$$
\begin{gathered}
\int\left(\frac{Q}{2}+y Q_{y}\right) y\left(\frac{Q}{2}+y Q_{y}\right)=0, \quad \int\left(Q_{y}\right) y\left(\frac{Q}{2}+y Q_{y}\right)=\int\left(\frac{Q}{2}+y Q_{y}\right)^{2} \neq 0, \\
\int\left(\frac{Q}{2}+y Q_{y}\right) y Q_{y}=\int\left(\frac{Q}{2}+y Q_{y}\right)^{2} \neq 0, \quad \int\left(Q_{y}\right) y Q_{y}=0,
\end{gathered}
$$

where we have used $\int Q\left(\frac{Q}{2}+y Q_{y}\right)=0$, and parity properties.

Note that by invariance of the equation by translation in time, we may assume that $t\left(u_{0}\right)=0$ in the rest of this paper. We also assume that $u(t, x)$ satisfies the decomposition of Lemma 1 , and not $-u(t, x)$. This is the first possibility in Theorem 1 .

Let

$$
s=\int_{0}^{t} \frac{d t^{\prime}}{\lambda^{3}\left(t^{\prime}\right)}, \quad \text { or equivalently, } \quad \frac{d s}{d t}=\frac{1}{\lambda^{3}} .
$$

Observe that by the scaling property of equation (1) and local wellposedness of (1) in $H^{1}$ (see introduction), we have by (14)

$$
\text { for all } t \in[0, T), \quad \lambda(t) \leq C(T-t)^{1 / 3} \text {. }
$$

Therefore, when $t$ takes its values on $[0, T), s$ takes its values in all $\mathbf{R}^{+}$. In the next lemma, following [11], and [15], Lemma 3 and Corollary 1, we gather useful properties of $\varepsilon(s), \lambda(s)$ and $x(s)$.

Lemma 2 (Properties of the decomposition). There exists $0<\alpha_{3} \leq \alpha_{2}$, such that if $\alpha_{0}<\alpha_{3}$ then $\lambda(s)$ and $x(s)$ are $C^{1}$ functions on $\mathbf{R}^{+}$and the following properties exist:

(i) Equation of $\varepsilon(s)$. The function $\varepsilon(s)$ satisfies, for $s \in \mathbf{R}^{+}, y \in \mathbf{R}$,

$$
\begin{aligned}
\varepsilon_{s}= & (L \varepsilon)_{y}+\frac{\lambda_{s}}{\lambda}\left(\frac{Q}{2}+y Q_{y}\right)+\left(\frac{x_{s}}{\lambda}-1\right) Q_{y} \\
& +\frac{\lambda_{s}}{\lambda}\left(\frac{\varepsilon}{2}+y \varepsilon_{y}\right)+\left(\frac{x_{s}}{\lambda}-1\right) \varepsilon_{y}-(R(\varepsilon))_{y},
\end{aligned}
$$

where $L \varepsilon=-\varepsilon_{x x}+\varepsilon-5 Q^{4} \varepsilon$ and $R(\varepsilon)=10 Q^{3} \varepsilon^{2}+10 Q^{2} \varepsilon^{3}+5 Q \varepsilon^{4}+\varepsilon^{5}$.

(ii) Smallness properties. For some $C>0$,

$$
\text { for all } s \geq 0, \quad|\varepsilon(s)|_{L^{2}}+\left|\varepsilon_{y}(s)\right|_{L^{2}} \leq C \sqrt{\alpha_{0}},
$$

$$
\text { for all } t \in[0, T), \quad \frac{\left|u_{x}(t)\right|_{L^{2}}}{2\left|Q_{x}\right|_{L^{2}}} \leq \frac{1}{\lambda(t)} \leq \frac{2\left|u_{x}(t)\right|_{L^{2}}}{\left|Q_{x}\right|_{L^{2}}}
$$

(iii) Control of the geometrical parameters. For some $C>0$,

$$
\text { for all } s \geq 0, \quad\left|\frac{\lambda_{s}}{\lambda}\right|+\left|\frac{x_{s}}{\lambda}-1\right| \leq C\left(\int \varepsilon^{2} e^{-\frac{|y|}{2}}\right)^{1 / 2} \leq C \sqrt{\alpha_{0}} .
$$


Proof. For (i) see [11], and [15]. Let us recall the following structural properties of $L$ :

$$
\begin{gathered}
L\left(Q^{3}\right)=-8 Q^{3}, \quad L\left(Q_{y}\right)=0 \\
\text { for all } \varepsilon \in H^{1}(\mathbf{R}) \text {, if } \int Q^{3} \varepsilon=\int Q_{y} \varepsilon=0 \text { then }(L \varepsilon, \varepsilon) \geq \int \varepsilon^{2} .
\end{gathered}
$$

We prove (ii) using some ideas from [15]. By the definition of $\varepsilon$ and the conservation of mass, we have

$$
2 \int Q \varepsilon+\int \varepsilon^{2}=\int u^{2}-\int Q^{2}=\int u_{0}^{2}-\int Q^{2}=\alpha_{0} .
$$

By the conservation of energy, we have $\lambda^{2} E(Q+\varepsilon)=E(u(t))=E_{0}<0$, and by direct calculations,

$$
\begin{aligned}
E(Q & +\varepsilon)+\left(\int Q \varepsilon+\frac{1}{2} \int \varepsilon^{2}\right) \\
& =\frac{1}{2}(L \varepsilon, \varepsilon)-\frac{1}{6}\left[20 \int Q^{3} \varepsilon^{3}+15 \int Q^{2} \varepsilon^{4}+6 \int Q \varepsilon^{5}+\int \varepsilon^{6}\right] .
\end{aligned}
$$

Therefore,

$$
(L \varepsilon, \varepsilon) \leq \alpha_{0}+C|\varepsilon|_{H^{1}}|\varepsilon|_{L^{2}}^{2} .
$$

Note that by the choice of orthogonality conditions on $\varepsilon$, this is not sufficient to conclude the proof of (ii) directly. Indeed, they are suitable for the Virial identity but not for the energy identity. Nevertheless, consider an auxiliary function $\varepsilon_{1}=\varepsilon-a\left(\frac{Q}{2}+y Q_{y}\right)-b Q_{y}$, where

$$
\int \varepsilon_{1} Q^{3}=\int \varepsilon_{1} Q_{y}=0
$$

and take $a=\frac{\int \varepsilon Q^{3}}{\int\left(\frac{Q}{2}+y Q_{y}\right) Q^{3}}, b=\frac{\int \varepsilon Q_{y}}{\int Q_{y}^{2}}$ (note that $\left.\int\left(\frac{Q}{2}+y Q_{y}\right) Q^{3}=\frac{1}{4} \int Q^{4} \not \equiv 0\right)$. Note that we also have $\varepsilon=\varepsilon_{1}+a\left(\frac{Q}{2}+y Q_{y}\right)+b Q_{y}$, so that by orthogonality conditions on $\varepsilon, a=\frac{\int \varepsilon_{1}\left(\frac{Q}{2}+y Q_{y}\right)}{\int\left(\frac{Q}{2}+y Q_{y}\right)^{2}}, b=\frac{\int \varepsilon_{1} y\left(\frac{Q}{2}+y Q_{y}\right)}{\int\left(\frac{Q}{2}+y Q_{y}\right)^{2}}$.

Now, since $\int\left(\frac{Q}{2}+y Q_{y}\right) Q=0, L\left(\frac{Q}{2}+y Q_{y}\right)=-2 Q, L Q_{y}=0$, we find after some elementary calculations:

$$
\int \varepsilon Q=\int \varepsilon_{1} Q, \quad(L \varepsilon, \varepsilon)=\left(L \varepsilon_{1}, \varepsilon_{1}\right)-4 a(\varepsilon, Q) .
$$

By the expressions for $a$ and $b$, we have for some constant $K$,

$$
\frac{1}{K}\left(\varepsilon_{1}, \varepsilon_{1}\right) \leq(\varepsilon, \varepsilon) \leq K\left(\varepsilon_{1}, \varepsilon_{1}\right)
$$


Thus, from (23) and (25),

$$
\frac{1}{K}(\varepsilon, \varepsilon) \leq\left(\varepsilon_{1}, \varepsilon_{1}\right) \leq\left(L \varepsilon_{1}, \varepsilon_{1}\right) \leq \alpha_{0}+4|a||(\varepsilon, Q)|+C|\varepsilon|_{L^{2}}^{2}|\varepsilon|_{H^{1}} .
$$

For $\alpha_{0}$ small, $|\varepsilon|_{H^{1}}$ and $|a|$ are small and from the conservation of mass, we have $2|(\varepsilon, Q)| \leq \alpha_{0}+\int \varepsilon^{2}$; thus

$$
\frac{1}{K}(\varepsilon, \varepsilon) \leq 2 \alpha_{0}+C|\varepsilon|_{L^{2}}^{2}\left(|a|+|\varepsilon|_{H^{1}}\right) \leq 2 \alpha_{0}+\frac{1}{2 K}|\varepsilon|_{L^{2}}^{2} .
$$

Therefore, $(\varepsilon, \varepsilon) \leq 4 K \alpha_{0}$ and from $(25),(L \varepsilon, \varepsilon) \leq C \alpha_{0}$. The conclusion then comes from the fact that $|\varepsilon|_{H^{1}}^{2} \leq(L \varepsilon, \varepsilon)+5 \int Q^{4} \varepsilon^{2} \leq(L \varepsilon, \varepsilon)+c(\varepsilon, \varepsilon)$.

For (iii) note that by multiplying the equation of $\varepsilon(s)$ by $y Q_{y}$ and then by $y\left(\frac{Q}{2}+y Q_{y}\right)$, using the decay property of $Q$ at infinity, we obtain

$$
\begin{aligned}
& \left.\mid \frac{\lambda_{s}}{\lambda}\left(\mu_{0}+\int\left(\frac{\varepsilon}{2}+y \varepsilon_{y}\right) y Q_{y}\right)\right)-\int \varepsilon L\left(\left(y Q_{y}\right)_{y}\right) \mid \\
& \leq C\left|\frac{x_{s}}{\lambda}-1\right|\left(\int \varepsilon^{2} e^{-\frac{|y|}{2}}\right)^{1 / 2}+C \int \varepsilon^{2} e^{-\frac{|y|}{2}},
\end{aligned}
$$

and

$$
\begin{gathered}
\left|\left(\frac{x_{s}}{\lambda}-1\right)\left(\mu_{0}+\int \varepsilon_{y} y\left(\frac{Q}{2}+y Q_{y}\right)\right)-\int \varepsilon L\left(\left(y\left(\frac{Q}{2}+y Q_{y}\right)\right)_{y}\right)\right| \\
\leq C\left|\frac{\lambda_{s}}{\lambda}\right|\left(\int \varepsilon^{2} e^{-\frac{|y|}{2}}\right)^{1 / 2}+C \int \varepsilon^{2} e^{-\frac{|y|}{2}}
\end{gathered}
$$

where $\mu_{0}=\int\left(\frac{Q}{2}+y Q_{y}\right)^{2}>0$. Therefore, for $\alpha_{3}$ small enough,

$$
\begin{gathered}
\left|\frac{\lambda_{s}}{\lambda}-\frac{1}{\mu_{0}} \int \varepsilon L\left(\left(y Q_{y}\right)_{y}\right)\right|+\left|\left(\frac{x_{s}}{\lambda}-1\right)-\frac{1}{\mu_{0}} \int \varepsilon L\left(\left(y\left(\frac{Q}{2}+y Q_{y}\right)\right)_{y}\right)\right| \\
\leq C\left(\left|\frac{\lambda_{s}}{\lambda}\right|+\left|\frac{x_{s}}{\lambda}-1\right|\right)\left(\int \varepsilon^{2} e^{-\frac{|y|}{2}}\right)^{1 / 2}+C \int \varepsilon^{2} e^{-\frac{|y|}{2}},
\end{gathered}
$$

and (21) follows. Note that we have in addition

$$
\begin{aligned}
\left|\frac{\lambda_{s}}{\lambda}-\frac{1}{\mu_{0}} \int \varepsilon L\left(\left(y Q_{y}\right)_{y}\right)\right| & +\left|\left(\frac{x_{s}}{\lambda}-1\right)-\frac{1}{\mu_{0}} \int \varepsilon L\left(\left(y\left(\frac{Q}{2}+y Q_{y}\right)\right)_{y}\right)\right| \\
& \leq C \int \varepsilon^{2}(s) e^{-\frac{|y|}{2}} .
\end{aligned}
$$

In the rest of this paper, $u(t)$ denotes a solution blowing up in finite or infinite time $T>0$ such that $\int u^{2}=\int Q^{2}+\alpha_{0}$, with $\alpha_{0}<\alpha_{3}$; we consider the decomposition in $\varepsilon, \lambda$ and $x$, satisfying the properties given in Lemmas 1 and 2 .

2.2. Relations. We consider four different quantities for $\varepsilon(s)$. Two of them are related to conservation laws, and the others concern dispersion relations respectively in $L^{1}$ and in $L^{2}$. 
LEMma 3 (Mass and energy conservation).

$$
\begin{gathered}
2 \int \varepsilon Q+\int \varepsilon^{2}=\alpha_{0} \quad(\text { mass conservation }) \\
\left|\lambda^{2} E_{0}+\int \varepsilon Q-\frac{1}{2} \int \varepsilon_{y}^{2}\right| \leq C \int \varepsilon^{2} e^{-|y|}+C \alpha_{0}^{2} \int \varepsilon_{y}^{2} \quad \text { (energy relation). }
\end{gathered}
$$

Proof. First, we recall that the conservation of the $L^{2}$ norm of $u(t)$ and the notation $\alpha_{0}=\int u^{2}-\int Q^{2}$ give (27) directly.

Second, by (24), the decay properties of $Q,|\varepsilon(s)|_{L^{\infty}} \leq C \sqrt{\alpha_{0}}$ (by (19)), and the Gagliardo-Nirenberg inequality $\int \varepsilon^{6} \leq C\left(\int \varepsilon^{2}\right)^{2} \int \varepsilon_{y}^{2} \leq C \alpha_{0}^{2} \int \varepsilon_{y}^{2}$, we obtain (28).

Next, defining

$$
J(s)=\left[\int \varepsilon(s, y)\left(\int_{y}^{+\infty}\left(\frac{Q}{2}+z Q_{z}\right) d z\right) d y\right]-\frac{1}{4}\left(\int Q\right)^{2},
$$

we have the following lemma.

Proposition 1 ( $L^{1}$-type dispersion). Assume that $J\left(s_{0}\right)$ is well-defined for some $s_{0}$. Then $J(s)$ is well-defined for all $s \geq 0$ and is of class $C^{1}$. Moreover,

$$
\left|J_{s}+\frac{\lambda_{s}}{2 \lambda} J+2 \int \varepsilon Q\right| \leq C \int \varepsilon^{2} e^{-\frac{|y|}{2}}
$$

Proof. We obtain the equation satisfied by $J_{s}$ and $J$ by multiplying the equation of $\varepsilon$ (see Lemma 2 (i)) by $\int_{y}^{+\infty} \frac{Q}{2}+z Q_{z}$ and then integrating by parts. This calculation is formal but can be justified rigorously by regularization arguments; see the proof of Lemma 6 in [11]. We obtain

$$
\begin{aligned}
J_{s}+\frac{\lambda_{s}}{2 \lambda} J+2 \int \varepsilon Q= & \frac{\lambda_{s}}{\lambda} \int y\left(\frac{Q}{2}+y Q_{y}\right) \varepsilon+\left(\frac{x_{s}}{\lambda}-1\right) \int\left(\frac{Q}{2}+y Q_{y}\right) \varepsilon \\
& -\int\left(\frac{Q}{2}+y Q_{y}\right) R(\varepsilon) .
\end{aligned}
$$

We have used in particular $L\left(\frac{Q}{2}+y Q_{y}\right)=-2 Q, \int Q\left(\frac{Q}{2}+y Q_{y}\right)=0$ and

$$
\int\left(\frac{Q}{2}+y Q_{y}\right) \int_{y}^{+\infty}\left(\frac{Q}{2}+z Q_{z}\right)=\frac{1}{2}\left(\int \frac{Q}{2}+y Q_{y}\right)^{2}=\frac{1}{8}\left(\int Q\right)^{2} .
$$

From Lemma 2, we have

$$
\left|\int R(\varepsilon)\left(\frac{Q}{2}+y Q_{y}\right)\right|+\left|\frac{\lambda_{s}}{\lambda}\right|^{2}+\left|\frac{x_{s}}{\lambda}-1\right|^{2} \leq C \int \varepsilon^{2} e^{-\frac{|y|}{2}},
$$


and

$$
\left(\int\left(\frac{Q}{2}+y Q_{y}\right) \varepsilon\right)^{2} \leq C\left(\int\left(|y| e^{-\left(1-\frac{1}{\sqrt{2}}\right)|y|}\right)\left(|\varepsilon| e^{-\frac{|y|}{\sqrt{2}}}\right)\right)^{2} \leq C \int \varepsilon^{2} e^{-\frac{|y|}{2}},
$$

which proves (29).

Finally, we will need a Virial type relation. In [11], we considered a quantity of the type $\int y \varepsilon^{2}$. Unfortunately, in our situation, we do not have good control on $\varepsilon$ on the right (i.e. as $y \rightarrow+\infty$ ). Therefore, we need a localized version of this identity, which is given in the next lemma.

Let $\Phi \in \mathcal{C}^{2}(\mathbf{R}), \Phi(x)=\Phi(-x), \Phi^{\prime} \leq 0$ on $\mathbf{R}^{+}$, such that

$$
\Phi(x)=1 \text { on }[0,1] ; \quad \Phi(x)=e^{-x} \text { on }[2,+\infty), \quad e^{-x} \leq \Phi(x) \leq 3 e^{-x} \text { on } \mathbf{R}^{+} .
$$

Let

$$
\Psi(x)=\int_{0}^{x} \Phi(y) d y .
$$

Note that $\Psi$ is an odd function, $\Psi(x)=x$ on $[-1,1]$, and $|\Psi(x)| \leq 3$ on $\mathbf{R}$.

For a parameter $A>0$, we set

$$
\Psi_{A}(x)=A \Psi\left(\frac{x}{A}\right), \quad \text { so that } \quad \Psi_{A}^{\prime}(x)=\Phi\left(\frac{x}{A}\right)=\Phi_{A}(x), \quad \text { and }
$$

$\Psi_{A}(x)=x$ on $[-A, A], \quad\left|\Psi_{A}(x)\right| \leq 3 A$ on $\mathbf{R}, \quad e^{-\frac{|x|}{A}} \leq \Phi_{A}(x) \leq 3 e^{-\frac{|x|}{A}}$ on $\mathbf{R}$.

Proposition 2 (Local Virial relation, $L^{2}$-type dispersion). $x$ There exists $A_{0}>2, \alpha_{5}>0$ and $\delta_{0}>0$ such that for $\alpha_{0}<\alpha_{5}$,

$$
\begin{aligned}
& \text { if for all } s \geq 0, \quad \int y Q_{y} \varepsilon(s)=\int y\left(\frac{Q}{2}+y Q_{y}\right) \varepsilon(s)=0, \text { then } \\
& \left(\int \Psi_{A_{0}} \varepsilon^{2}\right)_{s} \leq-\delta_{0} \int\left(\varepsilon^{2}+\varepsilon_{y}^{2}\right) e^{-\frac{|y|}{A_{0}}}+\frac{1}{\delta_{0}}\left(\int \varepsilon Q\right)^{2} .
\end{aligned}
$$

Proof. First we note that this Virial relation is reminiscent of the Virial identity for $\varepsilon$ established in [11]. Indeed, when $\int y \varepsilon^{2}$ is defined, we have from $[11$, Lemma 5]:

$$
\begin{aligned}
\left(\frac{1}{2} \int y \varepsilon^{2}\right)_{s}+ & \frac{\lambda_{s}}{\lambda} \frac{1}{2} \int y \varepsilon^{2} \\
= & \frac{\lambda_{s}}{\lambda} \int y\left(\frac{Q}{2}+y Q_{y}\right) \varepsilon+\left(\frac{x_{s}}{\lambda}-1\right)\left(\int y Q_{y} \varepsilon-\frac{1}{2} \int \varepsilon^{2}\right) \\
& -\frac{3}{2}(L \varepsilon, \varepsilon)+\int \varepsilon^{2}-10 \int Q^{3}\left(\frac{Q}{2}+y Q_{y}\right) \varepsilon^{2} \\
& +10 \int\left(\frac{2 Q^{3}}{3}-y Q^{2} Q_{y}\right) \varepsilon^{3}+5 \int\left(\frac{3 Q^{2}}{2}-y Q Q_{y}\right) \varepsilon^{4} \\
& +\int\left(4 Q-y Q_{y}\right) \varepsilon^{5}+\frac{5}{6} \int \varepsilon^{6} .
\end{aligned}
$$


This Virial identity is obtained formally by multiplying the equation of $\varepsilon$ by $y \varepsilon$, integrating by parts, and using some properties of $Q$ and the operator $L$. See Lemma 5 in [11] for a complete proof and regularizing argument. The formula above does not require any orthogonality conditions on $\varepsilon(s)$.

In [12], we established the following. Denote

$$
\begin{aligned}
H_{\infty}(\varepsilon, \varepsilon) & =\frac{3}{2}(L \varepsilon, \varepsilon)-\int \varepsilon^{2}+10 \int Q^{3}\left(\frac{Q}{2}+y Q_{y}\right) \varepsilon^{2} \\
& =\frac{3}{2} \int \varepsilon_{y}^{2}+\frac{1}{2} \int \varepsilon^{2}-\frac{5}{2} \int Q^{4} \varepsilon^{2}+10 \int y Q_{y} Q^{3} \varepsilon^{2}
\end{aligned}
$$

There exists $\delta_{1}>0$ such that

$$
\text { if } \int Q \varepsilon=\int y\left(\frac{Q}{2}+y Q_{y}\right) \varepsilon=0 \quad \text { then } \quad H_{\infty}(\varepsilon, \varepsilon) \geq \delta_{1} \int\left(\varepsilon_{y}^{2}+\varepsilon^{2}\right) \text {. }
$$

(See Proposition 4 in [12], and its proof. Note that this result is optimal.)

Therefore, if

$$
\int y Q_{y} \varepsilon=\int Q \varepsilon=\int y\left(\frac{Q}{2}+y Q_{y}\right) \varepsilon=0
$$

then by the Virial identity and the control on $\frac{\lambda_{s}}{\lambda}, \frac{x_{s}}{\lambda}-1$ and $|\varepsilon|_{L^{\infty}}$ given by Lemma 2 (ii), (iii), we obtain

$$
\left(\int y \varepsilon^{2}\right)_{s}+\frac{\lambda_{s}}{\lambda} \int y \varepsilon^{2} \leq-2 \delta_{1} \int\left(\varepsilon_{y}^{2}+\varepsilon^{2}\right)+C\left(\int \varepsilon^{2}\right)^{3 / 2} \leq-\delta_{1} \int\left(\varepsilon_{y}^{2}+\varepsilon^{2}\right),
$$

for $|\varepsilon|_{H^{1}}$ small.

In fact, the orthogonality condition $\int Q \varepsilon=0$ can be replaced by an additional term in the Virial relation : if only $\int y Q_{y} \varepsilon=\int y\left(\frac{Q}{2}+y Q_{y}\right) \varepsilon=0$ then

$$
\left(\int y \varepsilon^{2}\right)_{s}+\frac{\lambda_{s}}{\lambda} \int y \varepsilon^{2} \leq-\frac{\delta_{1}}{2} \int\left(\varepsilon_{y}^{2}+\varepsilon^{2}\right)+C\left(\int Q \varepsilon\right)^{2}
$$

for $|\varepsilon|_{H^{1}}$ small.

The proof of Proposition 2 is a local version of this result, seen as a perturbation of the previous identity (note that $\Psi_{A}$ is a cut-off of $y$ since $\Psi_{A}(y)=y$ on $\left.[-A, A]\right)$. Since the arguments are rather technical, we present the rest of the proof in Appendix A. 
2.3. Exponential decay on the left. Together with relations given in the previous subsections, we now introduce a fundamental tool which links $L^{2}$ concentration as a Dirac mass at the blow-up time (or $L^{2}$ compactness for large time) with exponential decay on the left. This technique was introduced in $[15]$.

Lemma 4 (Exponential decay in $L^{2}$ norm on the left). There exists $\alpha_{6}>0, C_{1}, C_{2}>0$ satisfying the following property. Suppose $\alpha_{0}<\alpha_{6}$. Suppose that $u^{2}(t)$ blows up as a Dirac mass at the blow-up time, in the sense that

$$
u^{2}(t, x+x(t)) \rightarrow\left(\int u_{0}^{2}\right) \delta_{x=0}, \quad \text { as } t \uparrow T .
$$

If $s_{1}<s_{2}$ satisfy

for all $s \geq s_{1}, \lambda(s) \leq \lambda\left(s_{1}\right)$, and for all $s \in\left[s_{1}, s_{2}\right], \quad \frac{\lambda\left(s_{1}\right)}{1.1} \leq \lambda(s) \leq \lambda\left(s_{1}\right)$,

then

$$
\text { for all } y_{0}<0, \text { for all } s \in\left[s_{1}, s_{2}\right], \quad \int_{y<y_{0}} \varepsilon^{2}(s) \leq C_{1} e^{-C_{2}\left|y_{0}\right|} \text {. }
$$

Note that as in [15], we need a control on the oscillations of $\left|u_{x}(t)\right|_{L^{2}}$ (or $\lambda(t)$ ) which allows blow up. As a direct consequence, we have:

Corollary 1 (Exponential decay in $L^{\infty}$ norm on the left). Under the assumptions of the preceding lemma,

$$
\text { for all } y<0, \text { for all } s \in\left[s_{1}, s_{2}\right], \quad|\varepsilon(s, y)| \leq C_{1}^{\prime} \alpha_{0}^{1 / 4} e^{-C_{2}^{\prime}|y|} .
$$

Proof of Corollary 1. From the proof of Gagliardo-Nirenberg inequality, and Lemmas 2 and 4, we have: for all $y_{0}<0$ and $s \in\left[s_{1}, s_{2}\right]$,

$$
\left|\varepsilon\left(s, y_{0}\right)\right| \leq\left(\int \varepsilon_{y}^{2}\right)^{1 / 4}\left(\int_{y<y_{0}} \varepsilon^{2}\right)^{1 / 4} \leq C_{1}^{\prime} \alpha_{0}^{1 / 4} e^{-C_{2}^{\prime}\left|y_{0}\right|} .
$$

The proof of Lemma 4 follows essentially from techniques introduced in [15] and [12]. We give some notation, and then we recall a result from [15] concerning the solutions of (1).

Let $K=2 \sqrt{3}$ (for example). Define

for all $x \in \mathbf{R}, \phi(x)=c Q\left(\frac{x}{K}\right), \psi(x)=\int_{-\infty}^{x} \phi(y) d y$, where $c=\frac{1}{K \int_{-\infty}^{+\infty} Q}$, so that

$$
\text { for all } x \in \mathbf{R}, \quad 0 \leq \psi(x) \leq 1, \lim _{x \rightarrow-\infty} \psi(x)=0, \quad \lim _{x \rightarrow+\infty} \psi(x)=1 .
$$


Consider $z(t)$ a solution of (1) satisfying the decomposition of $\S 2.1$, with parameters $\lambda_{z}$ and $x_{z}$. For $x_{0} \in \mathbf{R}$, and $t_{0} \geq 0$ define

for all $t \geq 0, \quad \mathcal{I}_{x_{0}, t_{0}}(t)=\int z^{2}(t, x) \psi\left(x-x_{z}\left(t_{0}\right)-x_{0}-\frac{1}{4}\left(x_{z}(t)-x_{z}\left(t_{0}\right)\right)\right) d x$.

LEMma 5 (Almost monotonicity of the mass on the left [15]). Suppose that for all $t \geq t_{0}, 0<\lambda_{z}(t) \leq 1.1$. There exist $\alpha_{6}>0, C_{0}>0$ and $a_{0}>0$ such that if $\alpha_{0}<\alpha_{6}$, then

for all $x_{0} \leq-a_{0}$, for all $t \geq t_{0}, \mathcal{I}_{x_{0}, t_{0}}(t)-\mathcal{I}_{x_{0}, t_{0}}\left(t_{0}\right) \leq C_{0} e^{\frac{x_{0}}{3}}$.

Sketch of the proof of Lemma 5 . Let $\widetilde{x}=x-x_{z}\left(t_{0}\right)-x_{0}-\frac{1}{4}\left(x_{z}(t)-x_{z}\left(t_{0}\right)\right)$. We have by direct calculations

$$
\begin{aligned}
\mathcal{I}_{x_{0}, t_{0}}^{\prime}(t)= & -3 \int z_{x}^{2}(t, x) \phi(\widetilde{x})-\frac{\left(x_{z}\right) t}{4} \int z^{2}(t, x) \phi(\widetilde{x}) \\
& +\int z^{2}(t, x) \phi^{\prime \prime}(\widetilde{x})+\frac{5}{3} \int z^{6}(t, x) \phi(\widetilde{x}),
\end{aligned}
$$

and $\phi^{\prime \prime} \leq \frac{1}{K^{2}} \phi, \frac{9}{10 \lambda_{z}^{2}} \leq\left(x_{z}\right)_{t}$. It follows from control of the last term of the expression of $\mathcal{I}_{x_{0}, t_{0}}^{\prime}(t)$, using the decomposition of the solution $z$, that

$$
\mathcal{I}_{x_{0}, t_{0}}^{\prime}(t) \leq C\left(x_{z}\right)_{t} e^{-\frac{3}{4 K}\left(x_{z}(t)-x_{z}\left(t_{0}\right)\right)+\frac{x_{0}}{K}} .
$$

Then the result follows by integration in time. See [15] for details.

Proof of Lemma 4. Let $s_{1}<s_{2}$ be as in the statement of Lemma 4, and let $s_{0} \in\left[s_{1}, s_{2}\right]$. We apply Lemma 5 to a rescaled version of $u(t)$.

Let $\lambda_{0}=\lambda\left(s_{0}\right)$, and

$$
z\left(t^{\prime}, x^{\prime}\right)=\lambda_{0}^{1 / 2} u\left(\lambda_{0}^{3} t^{\prime}, \lambda_{0} x^{\prime}\right) .
$$

The function $z$ is a solution of $(1)$. We note that $\lambda_{z}(s)=\lambda(s) / \lambda_{0}$ and $x_{z}(s)=$ $x(s) / \lambda_{0}$ are the geometrical parameters associated to $z$. Now

$$
\begin{gathered}
z^{2}\left(t^{\prime}, x^{\prime}+x_{z}\left(t^{\prime}\right)\right) \rightarrow\left(\int u_{0}^{2}\right) \delta_{x^{\prime}=0}, \quad \text { as } t^{\prime} \uparrow T / \lambda_{0}^{3}, \\
\quad \text { for all } s \geq s_{0}, \quad \lambda_{z}(s)=\frac{\lambda(s)}{\lambda\left(s_{0}\right)} \leq \frac{\lambda\left(s_{1}\right)}{\lambda\left(s_{0}\right)} \leq 1.1,
\end{gathered}
$$

since $s_{0} \in\left[s_{1}, s_{2}\right]$. Let $y_{0}<-a_{0}$ and let $s \geq s_{0}$. We apply Lemma 5 to $z$ between $s_{0}$ and $s \geq s_{0}$. We obtain

$$
\begin{aligned}
& \int z^{2}\left(s_{0}, x^{\prime}\right) \psi\left(x^{\prime}-x_{z}\left(s_{0}\right)-y_{0}\right) d x^{\prime} \\
& \quad \geq-C e^{\frac{y_{0}}{3}}+\int z^{2}\left(s, x^{\prime}\right) \psi\left(x^{\prime}-x_{z}\left(s_{0}\right)-y_{0}-\frac{1}{4}\left(x_{z}(s)-x_{z}\left(s_{0}\right)\right)\right) d x^{\prime} .
\end{aligned}
$$


Let $v(s, y)=\varepsilon(s, y)+Q(y)=\lambda^{1 / 2}(s) u(s, \lambda(s) y+x(s))$, so that $v\left(s_{0}, y\right)=$ $z\left(s_{0}, y+x_{z}\left(s_{0}\right)\right)$; the left-hand side of $(32)$ is

$$
\int v^{2}\left(s_{0}, y\right) \psi\left(y-y_{0}\right) d y \text {. }
$$

On the other hand, since $x_{z}(s) \geq x_{z}\left(s_{0}\right)$ (by $(21), \frac{d x_{z}}{d s} \geq 0$ ), and $\psi$ is nondecreasing, we have

$$
\int v^{2}\left(s_{0}, y\right) \psi\left(y-y_{0}\right) d y \geq-C e^{\frac{y_{0}}{3}}+\int z^{2}\left(s, x^{\prime}\right) \psi\left(x^{\prime}-x_{z}(s)-y_{0}\right) d x^{\prime},
$$

which gives, as $s \rightarrow+\infty$ (which corresponds to $t^{\prime} \rightarrow T / \lambda_{0}^{3}$ ) ,

$$
\int v^{2}\left(s_{0}, y\right) \psi\left(y-y_{0}\right) d y \geq-C e^{\frac{y_{0}}{3}}+\left(\int u_{0}^{2}\right) \psi\left(-y_{0}\right) .
$$

Now we use the properties of the function $\psi$ and $\int v^{2}\left(s_{0}\right)=\int u_{0}^{2}$. Therefore,

$$
\begin{aligned}
\int_{y<y_{0}} v^{2}\left(s_{0}, y\right) d y & \leq 2 \int_{y<y_{0}} v^{2}\left(s_{0}, y\right)\left(1-\psi\left(y-y_{0}\right)\right) d y \\
& \leq 2 \int v^{2}\left(s_{0}, y\right)\left(1-\psi\left(y-y_{0}\right)\right) d y \\
& \leq 2\left[\int u_{0}^{2}+C e^{\frac{y_{0}}{3}}-\psi\left(-y_{0}\right) \int u_{0}^{2}\right] \\
& \leq C\left[e^{\frac{y_{0}}{3}}+\left(1-\psi\left(-y_{0}\right)\right)\right] \leq C e^{c^{\prime} y_{0}}
\end{aligned}
$$

The proof of the lemma then follows from $\varepsilon\left(s_{1}, y\right)=v\left(s_{1}, y\right)-Q(y)$, and the decay properties of $Q$. Note that $|\varepsilon(s)|_{L^{\infty}} \leq C \sqrt{\alpha_{0}}$ (by Gagliardo-Nirenberg inequality and Lemma 2) extends the inequality for all $y<0$.

\section{Direct proof of a lower bound at blow-up time}

In this section, we establish a result simpler that Theorems 1 and 2 . We prove that for $\alpha_{0}$ small, there is no solution $u(t)$, blowing up in some finite time $T>0$ such that

$$
\exists C_{2}=C_{2}\left(u_{0}\right), \text { for all } t \in[0, T), \quad \int u_{x}^{2}(t) \leq \frac{C_{2}}{(T-t)^{2 / 3}}
$$

(note that there is no a priori control on $C_{2}$ ). Equivalently, for all solutions blowing up in finite time $T>0$, we have

$$
\limsup _{t \uparrow T}(T-t)^{2 / 3} \int u_{x}^{2}(t)=+\infty
$$

The proof is somewhat pedagogical and is based on some geometrical and scaling considerations to reduce the initial problem to an asymptotic problem; 
see $\S 3.1$. The fact that the asymptotic problem has no solution is then proved in $§ 3.2$. This proof of nonexistence, together with the Liouville theorem in [12] will be the base of the proof of Theorem 1. Nevertheless, we point out that the proof of (33) does not rely on the Liouville theorem.

3.1. Reduction to a solution blowing up as a Dirac mass in $L^{2}$. We prove (33) by contradiction. Assume that $u(t)$ blows up in finite time $T>0$ and satisfies

$$
\text { for all } t \in[0, T), \quad \int u_{x}^{2}(t) \leq \frac{C_{2}\left(u_{0}\right)}{(T-t)^{2 / 3}} \text {. }
$$

From this assumption, we construct another solution with the same properties as $u(t)$ and blowing up as a Dirac mass in $L^{2}$. This is obtained as a recurrent object as $t \uparrow T$.

Proposition 3 (Reduction to a solution blowing up as a Dirac mass in $L^{2}$ ). For $\alpha_{0}>0$ small enough, under the preceding assumptions, there exists a solution $\widetilde{u}(t)$ defined on $[0,1)$ such that

(i) $\int \widetilde{u}^{2}(t) \leq \int Q^{2}+\alpha_{0}$,

(ii) $E(\widetilde{u}) \leq 0$,

(iii) $\widetilde{u}(t)$ blows up at $t=1$, and for all $t \in[0,1),\left|\widetilde{u}_{x}(t)\right|_{L^{2}} \leq \frac{C}{(1-t)^{1 / 3}}$,

(iv) $\widetilde{u}^{2}(t, x+\widetilde{x}(t)) \rightarrow\left(\int \widetilde{u}^{2}(0)\right) \delta_{x=0}$, as $t \uparrow 1$.

Proof. Recall that from the assumption on $u(t)$, the introduction and (20), we have for all $t \in[0, T)$,

$$
\frac{C_{1}}{(T-t)^{2 / 3}} \leq \int u_{x}^{2}(t) \leq \frac{C_{2}}{(T-t)^{2 / 3}} \quad \text { and } \quad \frac{C_{1}^{\prime}}{(T-t)^{1 / 3}} \leq \frac{1}{\lambda(t)} \leq \frac{C_{2}^{\prime}}{(T-t)^{1 / 3}} .
$$

Let $\left(t_{n}\right)$ be an increasing sequence of $[0, T)$ such that $t_{n} \rightarrow T$ as $n \rightarrow+\infty$. For $t \in\left[1-T \lambda_{n}^{-3}, 1\right), x \in \mathbf{R}$, we set

$$
u_{n}(t, x)=\lambda_{n}^{1 / 2} u\left(\lambda_{n}^{3}(t-1)+T, \lambda_{n} x\right), \quad \text { where } \quad \lambda_{n}=\left(T-t_{n}\right)^{1 / 3} .
$$

For all $n, u_{n}$ is a solution of $(1)$ on $\left[1-T \lambda_{n}^{-3}, 1\right)$, blowing up at $t=1$, with initial data

$$
u_{n}(0, x)=\lambda_{n}^{1 / 2} u\left(t_{n}, \lambda_{n} x\right)
$$

and such that

(i) $\left|u_{n}\right|_{L^{2}}=|u|_{L^{2}},\left|u_{n}(0)\right|_{H^{1}} \leq C(C$ independent of $n)$,

(ii) $E\left(u_{n}\right) \rightarrow 0$, as $n \rightarrow+\infty$,

(iii) for all $t \in\left[1-T \lambda_{n}^{-3}, 1\right), \quad \frac{C_{1}}{(1-t)^{2 / 3}} \leq \int\left(u_{n}\right)_{x}^{2}(t, x) d x \leq \frac{C_{2}}{(1-t)^{2 / 3}}$. 


$$
\frac{C_{1}}{(1-t)^{2 / 3}}=\frac{C_{1} \lambda_{n}^{2}}{\left(\lambda_{n}^{3}(1-t)\right)^{2 / 3}} \leq \int\left(u_{n}\right)_{x}^{2}(t, x) d x \leq \frac{C_{2} \lambda_{n}^{2}}{\left(\lambda_{n}^{3}(1-t)\right)^{2 / 3}}=\frac{C_{2}}{(1-t)^{2 / 3}},
$$

applied to $t=0$. (ii) follows from $E\left(u_{n}\right)=\lambda_{n}^{2} E(u) \rightarrow 0$ as $n \rightarrow+\infty$.

Step 1. Definition and first properties of the limit object. We claim that there exists $\widetilde{u}(0) \in H^{1}(\mathbf{R}), \widetilde{u}(0) \not \equiv 0$, such that $0 \leq \alpha(\widetilde{u}(0)) \leq \alpha_{0}, E(\widetilde{u}(0)) \leq 0$, and there exists a subsequence of $\left(u_{n}\right)$, still denoted by $\left(u_{n}\right)$, satisfying

$$
u_{n}(0) \rightarrow \widetilde{u}(0) \quad \text { in } H^{1}(\mathbf{R}) .
$$

The existence of a subsequence of $\left(u_{n}(0)\right)$ which converges to some $\widetilde{u}(0) \in$ $H^{1}(\mathbf{R})$ weakly in $H^{1}(\mathbf{R})$ is a consequence of the uniform bound (i) on $\left(u_{n}(0)\right)$ in $H^{1}$.

Now, let us show that $\widetilde{u}(0) \not \equiv 0$. We first note that from (21) we have, for $\alpha_{0}$ small enough, $\left|\frac{x_{s}}{\lambda}-1\right|=\left|\lambda^{2} x_{t}-1\right| \leq 1 / 2$, and so

$$
\text { for all } t \in[0, T), \quad 0 \leq x_{t}(t) \leq \frac{3}{2 \lambda^{2}(t)} \leq \frac{C}{(T-t)^{2 / 3}},
$$

and so $x(t)$ approaches a limit as $t \uparrow T$, denoted $x(T)$. By considering $u(t, x+$ $x(T)$ ), solution of (1) instead of $u(t, x)$, we assume that

$x(T)=0$ and so, by integration, for all $t \in[0, T),|x(t)| \leq C(T-t)^{1 / 3} \leq C \lambda(t)$.

Now, by Lemma $1,\left|Q-\lambda^{1 / 2}\left(t_{n}\right) u\left(t_{n}, \lambda\left(t_{n}\right) x+x\left(t_{n}\right)\right)\right|_{H^{1}} \leq \delta^{\prime}\left(\alpha_{0}\right)$, and so, by scaling invariance of the $L^{2}$-norm,

$$
\left|u_{n}(0, x)-\left(\frac{\lambda_{n}}{\lambda\left(t_{n}\right)}\right)^{1 / 2} Q\left(\frac{\lambda_{n}}{\lambda\left(t_{n}\right)} x-\frac{x\left(t_{n}\right)}{\lambda\left(t_{n}\right)}\right)\right|_{L^{2}} \leq \delta^{\prime}\left(\alpha_{0}\right) .
$$

Using $\frac{\left|x\left(t_{n}\right)\right|}{\lambda\left(t_{n}\right)} \leq C$ and $C_{1}^{\prime} \leq \frac{\lambda_{n}}{\lambda\left(t_{n}\right)} \leq C_{2}^{\prime}$, there exists $A$ such that

$$
\left|u_{n}(0)\right|_{L^{2}(|x|>A)} \leq 2 \delta^{\prime}\left(\alpha_{0}\right),
$$

which implies for $\alpha_{0}$ small enough that

$$
\left|u_{n}(0)\right|_{L^{2}(|x|<A)} \geq|Q|_{L^{2}}-\frac{1}{4}|Q|_{L^{2}}=\frac{3}{4}|Q|_{L^{2}},
$$

and thus $|\widetilde{u}(0)|_{L^{2}} \geq \frac{3}{4}|Q|_{L^{2}}$, by strong convergence $L_{\text {loc }}^{2}$.

Next, note that by the properties of weak convergence, $0 \leq \alpha(\widetilde{u}(0))$ $\leq \alpha_{0}$ and $E(\widetilde{u}(0)) \leq 0$. The second fact is not obvious, and we prove it briefly.

Let us define a function $\rho$ such that

$$
0 \leq \rho \leq 1, \rho(x)=1 \text { for }|x| \leq 1, \rho(x)=0 \text { for }|x| \geq 2, \sqrt{\rho}, \sqrt{1-\rho} \in \mathcal{C}^{2},
$$

and for $k \in \mathbf{N}, \rho_{k}(x)=\rho\left(\frac{x}{k}\right)$. First, by direct calculations,

$$
E\left(u_{n}(0)\right)=E\left(u_{n}(0) \sqrt{\rho_{k}}\right)+E\left(u_{n}(0) \sqrt{1-\rho_{k}}\right)+R_{n, k},
$$


where $R_{n, k}=-\frac{1}{8} \int u_{n}^{2}(0)\left(\rho_{k}^{\prime}\right)^{2}\left(\frac{1}{\rho_{k}}+\frac{1}{1-\rho_{k}}\right)+\frac{1}{2} \int u_{n}^{6}(0) \rho_{k}\left(\rho_{k}-1\right)$ so that $R_{n, k} \rightarrow$ $R_{k}=-\frac{1}{8} \int \widetilde{u}^{2}(0)\left(\rho_{k}^{\prime}\right)^{2}\left(\frac{1}{\rho_{k}}+\frac{1}{1-\rho_{k}}\right)+\frac{1}{2} \int \widetilde{u}^{6}(0) \rho_{k}\left(\rho_{k}-1\right)$ as $n \rightarrow+\infty$, by the Rellich compactness theorem.

Second, observe that, for all $n,\left|u_{n}(0)-Q\right|_{L^{2}} \leq \frac{1}{2}|Q|_{L^{2}}$, and so, there exists $k_{0}>0$ such that for all $k>k_{0}$ and for all $n$, we have $\left|u_{n}(0) \sqrt{1-\rho_{k}}\right|_{L^{2}} \leq|Q|_{L^{2}}$. Therefore, by the Gagliardo-Nirenberg inequality (6), we have $E\left(u_{n}(0) \sqrt{1-\rho_{k}}\right)$ $\geq 0$.

Passing to the limit as $n \rightarrow+\infty$, since $E\left(u_{n}(0)\right) \rightarrow 0$, we obtain

$$
\varlimsup_{n \rightarrow \infty} E\left(u_{n}(0) \sqrt{\rho_{k}}\right)+R_{k} \leq 0 .
$$

But $\varlimsup_{n \rightarrow \infty} E\left(u_{n}(0) \sqrt{\rho_{k}}\right) \geq E\left(\widetilde{u}(0) \sqrt{\rho_{k}}\right)$, so that $E\left(\widetilde{u}(0) \sqrt{\rho_{k}}\right)+R_{k} \leq 0$. Finally, letting $k \rightarrow+\infty$, we obtain $E(\widetilde{u}(0)) \leq 0$.

Now, we consider the solution $\widetilde{u}(t)$ of (1) with initial data $\widetilde{u}(0)$, defined on $\left[0, T^{*}\right)$, for $T^{*}>0$. Since $E(\widetilde{u}(0)) \leq 0$ and $\alpha(\widetilde{u}(0)) \leq \alpha_{0}, \widetilde{u}(t)$ admits a canonical decomposition as in $\S 2.1$. We denote by $\widetilde{\lambda}(t), \widetilde{x}(t)$ and $\widetilde{\varepsilon}(t)$ the parameters of the decomposition.

Step 2. Properties of the limit solution. We consider the asymptotic object $\widetilde{u}(t)$ instead of the original solution $u(t)$ because $\widetilde{u}(t)$ satisfies more properties. Indeed, we prove that $\widetilde{u}^{2}(t)$ concentrates as a Dirac mass at the blow-up time. We claim the following result:

Proposition 4 (The asymptotic solution blows up as a Dirac mass in $L^{2}$ ). (a) $T^{*}=1$ and for all $t \in[0,1), \frac{C_{1}}{(1-t)^{2 / 3}} \leq \int \widetilde{u}_{x}^{2}(t) \leq \frac{C_{2}}{(1-t)^{2 / 3}}$.

(b) $\widetilde{u}^{2}(t, x+\widetilde{x}(t)) \rightarrow\left(\int \widetilde{u}^{2}(0)\right) \delta_{x=0}$ as $t \uparrow 1$.

Proof of (a). Let us recall the following result of stability of the weak convergence for the $\mathrm{KdV}$ equation.

$$
\begin{aligned}
& \text { Lemma } 6([12]) . \quad \text { For all } 0<t_{0}<\min \left(1, T^{*}\right) \text {, } \\
& \qquad \begin{array}{l}
\text { for all } t \in\left[0, t_{0}\right], \quad u_{n}(t) \rightarrow \widetilde{u}(t) \quad \text { in } H^{1}(\mathbf{R}), \\
u_{n} \rightarrow \widetilde{u} \quad \text { in } C\left(\left[0, t_{0}\right], L_{\text {loc }}^{2}(\mathbf{R})\right), \quad \lambda_{n} \rightarrow \widetilde{\lambda} \quad \text { in } C\left(\left[0, t_{0}\right], \mathbf{R}\right) .
\end{array}
\end{aligned}
$$

Proof. See Lemma 8 and Appendix D of [12]. Note that growth in $H^{1}$ can be seen in $L_{\text {loc }}^{2}$ norm by variational arguments.

We have from (iii), (20) and the preceding lemma,

$$
\text { for all } t \in\left[0, \min \left(1, T^{*}\right)\right), \quad \frac{C_{1}^{\prime}}{(1-t)^{1 / 3}} \leq \frac{1}{\widetilde{\lambda}(t)} \leq \frac{C_{2}^{\prime}}{(1-t)^{1 / 3}} \text {. }
$$


Thus, from (20),

$$
\text { for all } t \in\left[0, \min \left(1, T^{*}\right)\right), \quad \frac{C_{1}}{(1-t)^{2 / 3}} \leq \int \widetilde{u}_{x}^{2}(t) \leq \frac{C_{2}}{(1-t)^{2 / 3}} ;
$$

in particular $T^{*}=1$, and (a) is proved.

Proof of (b). (b) will be a consequence of

for all $A>0$, for all $t \in(0,1), \int_{|x|<A(1-t)^{1 / 3}} \widetilde{u}^{2}(t, x) d x \geq \int \widetilde{u}^{2}(0, x) d x-\frac{C}{A}-\frac{C}{A^{3}}$.

To prove (37), let us first note that from uniform estimates on $u_{n}$, we have

Claim. For all $B>0, n>0$, and $t_{1}, t_{2} \in\left[1-T \lambda_{n}^{-3}, 1\right)$,

$$
\int_{|x|<B} u_{n}^{2}\left(t_{2}\right) \geq \int_{|x|<B / 2} u_{n}^{2}\left(t_{1}\right)-C \frac{\left|t_{2}-t_{1}\right|^{1 / 3}}{B}-C \frac{\left|t_{2}-t_{1}\right|}{B^{3}} .
$$

Proof of claim (38). Let $\varphi: \mathbf{R} \rightarrow[0,1]$ be a smooth function such that $\varphi(x)=1$ for $|x| \leq 1 / 2$, and $\varphi(x)=0$ for $|x| \geq 1$. For $B>0$, we set $\varphi_{B}(x)=\varphi(x / B)$.

Now,

$$
\begin{aligned}
\frac{d}{d t} \int u_{n}^{2}(t) \varphi_{B}(x) d x= & -3 \int\left(u_{n}\right)_{x}^{2}(t) \varphi_{B}^{\prime}+\int u_{n}^{2}(t) \varphi_{B}^{(3)}+\frac{5}{3} \int u_{n}^{6}(t) \varphi_{B}^{\prime} \\
= & -\frac{3}{B} \int\left(u_{n}\right)_{x}^{2}(t) \varphi^{\prime}(x / B)+\frac{1}{B^{3}} \int u_{n}^{2}(t) \varphi^{(3)}(x / B) \\
& +\frac{5}{3 B} \int u_{n}^{6}(t) \varphi^{\prime}(x / B) .
\end{aligned}
$$

Therefore, by conservation of the $L^{2}$ norm and the Gagliardo-Nirenberg inequality,

$$
\begin{aligned}
\left|\frac{d}{d t} \int u^{2}(t) \varphi_{B}(x) d x\right| & \leq \frac{C}{B} \int u_{x}^{2}(t)+\frac{C}{B^{3}}+\frac{C}{B} \int u^{6}(t)\left|\varphi^{\prime}(x / B)\right| \\
& \leq \frac{C}{B^{3}}+\frac{C}{B} \frac{1}{(1-t)^{2 / 3}}
\end{aligned}
$$

By integration, we obtain

$$
\begin{aligned}
\int u^{2}\left(t_{1}\right) \varphi_{B}-\int u^{2}\left(t_{2}\right) \varphi_{B} & \leq \frac{C\left|t_{2}-t_{1}\right|}{B^{3}}+\frac{C}{B}\left|\int_{t_{1}}^{t_{2}} \frac{d t}{(1-t)^{2 / 3}}\right| \\
& \leq \frac{C\left|t_{2}-t_{1}\right|}{B^{3}}+\frac{C}{B}\left|\int_{t_{1}}^{t_{2}} \frac{d t}{\left(t_{2}-t\right)^{2 / 3}}\right| \\
& \leq C \frac{\left|t_{2}-t_{1}\right|}{B^{3}}+C \frac{\left|t_{2}-t_{1}\right|^{1 / 3}}{B} .
\end{aligned}
$$


Then claim (38) follows from

$$
\int u^{2}\left(t_{1}\right) \varphi_{B} \geq \int_{|x|<B / 2} u^{2}\left(t_{1}\right) \quad \text { and } \quad \int u^{2}\left(t_{2}\right) \varphi_{B} \leq \int_{|x|<B} u^{2}\left(t_{2}\right) .
$$

Now, we prove (37), using the fact that $\widetilde{u}$ is recurrent in $u$. Let $A>0$, and $t \in[0,1)$. Recall that $\lambda_{n}=\left(1-t_{n}\right)^{1 / 3}$, and $u_{n}(t, x)=\lambda_{n}^{1 / 2} u\left(\lambda_{n}^{3}(t-1)+T, \lambda_{n} x\right)$. When $m \in \mathbf{N}$, we have $u(t, x)=\frac{1}{\lambda_{m+n}^{1 / 2}} u_{m+n}\left(\frac{t-T}{\lambda_{m+n}^{3}}+1, \frac{x}{\lambda_{m+n}}\right)$, and so

$$
u_{n}(t, x)=\frac{\lambda_{n}^{1 / 2}}{\lambda_{m+n}^{1 / 2}} u_{m+n}\left(\frac{\lambda_{n}^{3}}{\lambda_{m+n}^{3}}(t-1)+1, \frac{\lambda_{n}}{\lambda_{m+n}} x\right) .
$$

By change of variable $y=\frac{\lambda_{n}}{\lambda_{m+n}} x$,

$\int_{|x|<A(1-t)^{1 / 3}} u_{n}^{2}(t, x) d x=\int_{|y|<\frac{\lambda_{n}}{\lambda_{m+n}} A(1-t)^{1 / 3}} u_{m+n}^{2}\left(\frac{\lambda_{n}^{3}}{\lambda_{m+n}^{3}}(t-1)+1, y\right) d y$.

Apply claim (38) to the solution $u_{m+n}$ with $t_{2}=\frac{\lambda_{n}^{3}}{\lambda_{m+n}^{3}}(t-1)+1, t_{1}=0$ and $B=\frac{\lambda_{n}}{\lambda_{m+n}} A(1-t)^{1 / 3}$ :

$$
\begin{aligned}
\int_{|y|<\frac{\lambda n}{\lambda_{m+n}} A(1-t)^{1 / 3}} & u_{m+n}^{2}\left(\frac{\lambda_{n}^{3}}{\lambda_{m+n}^{3}}(t-1)+1\right) \\
\geq & \int_{|y|<\frac{1}{2} \frac{\lambda_{n}}{\lambda_{m+n}} A(1-t)^{1 / 3}} u_{m+n}^{2}(0) \\
& -C \frac{\left|\frac{\lambda_{n}^{3}}{\lambda_{m+n}^{3}}(t-1)+1\right|^{1 / 3}}{\frac{\lambda_{n}}{\lambda_{m+n}} A(1-t)^{1 / 3}}-C \frac{\left|\frac{\lambda_{n}^{3}}{\lambda_{m+n}^{3}}(t-1)+1\right|}{\left(\frac{\lambda_{n}}{\lambda_{m+n}} A(1-t)^{1 / 3}\right)^{3}} .
\end{aligned}
$$

Now, let

- $X_{0}>0$ be such that $\int_{|x|>X_{0}} \widetilde{u}^{2}(0) \leq \frac{1}{A}$,

- $n>0$ (depending on $A, t$ and $X_{0}$ ) be such that

$$
\int_{|x|<A(1-t)^{1 / 3}} \widetilde{u}^{2}(t) \geq \int_{|x|<A(1-t)^{1 / 3}} u_{n}^{2}(t)-\frac{1}{A},
$$

and

$$
\text { for all } k \geq 0, \quad \int_{|x|<X_{0}} u_{k+n}^{2}(0) \geq \int_{|x|<X_{0}} \widetilde{u}^{2}(0)-\frac{1}{A},
$$

- $m$ be such that

$$
\frac{1}{2} \frac{\lambda_{n}}{\lambda_{m+n}} A(1-t)^{1 / 3}>X_{0} \quad \text { and } \quad \frac{\lambda_{m+n}^{3}}{\lambda_{n}^{3}}<1-t .
$$


Then, we obtain

$$
\frac{\left|\frac{\lambda_{n}^{3}}{\lambda_{m+n}^{3}}(t-1)+1\right|}{\left(\frac{\lambda_{n}}{\lambda_{m+n}} A(1-t)^{1 / 3}\right)^{3}}=\frac{(1-t) \frac{\lambda_{n}^{3}}{\lambda_{m+n}^{3}}-1}{\left(\frac{\lambda_{n}}{\lambda_{m+n}} A(1-t)^{1 / 3}\right)^{3}} \leq \frac{1}{A^{3}} .
$$

Therefore,

$$
\begin{aligned}
\int_{|x|<A(1-t)^{1 / 3}} \widetilde{u}^{2}(t) & \geq \int_{|x|<A(1-t)^{1 / 3}} u_{n}^{2}(t)-\frac{1}{A} \\
& \geq \int_{|x|<X_{0}} u_{m+n}^{2}(0)-\frac{C}{A^{3}}-\frac{C+1}{A} \\
& \geq \int \widetilde{u}^{2}(0)-\frac{C}{A^{3}}-\frac{C+3}{A} ;
\end{aligned}
$$

thus (37) holds.

Now, we finish the proof of (b). From (37), we have directly

$$
\widetilde{u}^{2}(t, x) \rightarrow\left(\int \widetilde{u}^{2}(0)\right) \delta_{x=0}, \quad \text { as } t \uparrow 1 .
$$

From the fact that $|\widetilde{u}(t)|_{L^{2}} \leq \frac{C}{(1-t)^{1 / 3}}$, as in Step $1, \widetilde{x}(t)$ has a limit as $t \uparrow 1$, denoted $\widetilde{x}(1)$. By $(39)$, we have $\widetilde{x}(1)=0$. Therefore,

$$
\widetilde{u}^{2}(t, x+\widetilde{x}(t)) \rightarrow\left(\int \widetilde{u}^{2}(0)\right) \delta_{x=0}, \quad \text { as } t \uparrow 1 .
$$

Thus Propositions 4 and 3 are proved.

In conclusion, for $\alpha_{0}$ small enough, from Proposition 3, Lemma 4 and Corollary 1 , since $\widetilde{\lambda}(t) \rightarrow 0$ as $t \uparrow T$, we obtain a solution $\widetilde{u}(t)$ on $[0,1)$, such that

(i) $\int \widetilde{u}^{2} \leq \int Q^{2}+\alpha_{0}$,

(ii) $E(\widetilde{u}) \leq 0$,

(iii) there exists $s_{1}<s_{2}$ satisfying

$$
\begin{aligned}
& \text { for all } s \geq s_{1}, \tilde{\lambda}(s) \leq \widetilde{\lambda}\left(s_{1}\right), \tilde{\lambda}\left(s_{2}\right)=\frac{\widetilde{\lambda}\left(s_{1}\right)}{1.1}, \\
& \text { for all } s \in\left[s_{1}, s_{2}\right], \frac{\tilde{\lambda}\left(s_{1}\right)}{1.1} \leq \tilde{\lambda}(s) \leq \widetilde{\lambda}\left(s_{1}\right)
\end{aligned}
$$

and

$$
\text { for all } y<0 \text {, for all } s \in\left[s_{1}, s_{2}\right],|\widetilde{\varepsilon}(s, y)| \leq C_{1} \alpha_{0}^{1 / 4} e^{-C_{2}|y|} .
$$
follows.

In the next subsection, we prove the nonexistence of such $\widetilde{u}(t)$, and $(33)$

3.2. Nonexistence of a focusing solution with exponential decay on the left. In this section, we prove the following proposition, which is crucial for the proof of the stability of $Q$ as a blow-up profile. 
Proposition 5 (Nonexistence of a focusing solution with exponential decay). There exists $\alpha_{I}>0$ such that there exists no solution $u(t)$ of (1) satisfying

(i) $\int u^{2} \leq \int Q^{2}+\alpha_{I}$,

(ii) $E(u) \leq 0$,

(iii) There exist $s_{1}<s_{2}$ such that

$$
\begin{aligned}
& \text { for all } s>s_{1}, \lambda(s) \leq \lambda\left(s_{1}\right), \lambda\left(s_{2}\right)=\frac{\lambda\left(s_{1}\right)}{1.1}, \\
& \text { for all } s \in\left[s_{1}, s_{2}\right], \frac{\lambda\left(s_{1}\right)}{1.1} \leq \lambda(s) \leq \lambda\left(s_{1}\right),
\end{aligned}
$$

(iv) $\varepsilon(s)$ is such that

$$
\text { for all } y<0, \text { for all } s \in\left[s_{1}, s_{2}\right], \quad|\varepsilon(s, y)| \leq C_{1} \alpha_{0}^{1 / 4} e^{-C_{2}|y|} .
$$

Proof. The proof proceeds in three steps. In the first step, we give the main argument assuming that two fundamental inequalities hold. In steps 2 and 3 , we prove these two inequalities. We work on the time interval $\left[s_{1}, s_{2}\right]$.

Step 1. The main argument. The problem in this proof is to understand the size of different quantities such as

$$
\int_{s_{1}}^{s_{2}} \int \varepsilon^{2}, \quad \int_{s_{1}}^{s_{2}} \int \varepsilon_{y}^{2}, \quad \int_{s_{1}}^{s_{2}} \int \varepsilon^{2} e^{-\frac{|y|}{2}}
$$

(and also at some point $\int_{s_{1}}^{s_{2}} \int \varepsilon Q$.) Two difficulties are that we have no control of the size of the interval $\left[s_{1}, s_{2}\right]$ (the "doubling time") in terms of $\alpha_{0}$, and that the terms we integrate in time are oscillatory integrals.

We claim that there exists $\alpha_{7}>0$ such that if $\alpha_{0}<\alpha_{7}$ then the following two inequalities hold:

- There exists $C_{I}>0$ (independent of $\alpha_{0}$ ) such that

$$
C_{I} \int_{s_{1}}^{s_{2}} \int \varepsilon^{2} e^{-\frac{|y|}{2}} \geq 1+\int_{s_{1}}^{s_{2}} \int \varepsilon_{y}^{2}+\left|E_{0}\right| \int_{s_{1}}^{s_{2}} \lambda^{2} .
$$

This is a consequence of a dispersion relation in $L^{1}$ in terms of $J(s)$ (Proposition 1) and exponential decay. See step 2 for the proof.

- Let $A_{0}>2$ be as in Proposition 2. There exists $C_{I I}>0$ (independent of $\left.\alpha_{0}\right)$ such that

$$
\int_{s_{1}}^{s_{2}} \int \varepsilon^{2} e^{-\frac{|y|}{A_{0}}} \leq C_{I I} \alpha_{0}^{1 / 2}\left[1+\int_{s_{1}}^{s_{2}} \int \varepsilon_{y}^{2}+\left|E_{0}\right| \int_{s_{1}}^{s_{2}} \lambda^{2}\right] .
$$

This is a consequence of the local Virial type relation (Proposition 2), i.e. $L^{2}$ dispersion. See step 3 for the proof. 
Note that since $A_{0}>2$, the contradiction is obvious for $\alpha_{0}<\left(\frac{1}{C_{I} C_{I I}}\right)^{2}$. Thus we need only prove (42) and (43).

Step 2. Proof of (42). Recall that we have the following definition of $J(s)$ :

$$
J(s)=\int \varepsilon(s, y) \int_{y}^{+\infty}\left(\frac{Q}{2}+z Q_{z}\right)-\frac{1}{4}\left(\int Q\right)^{2} .
$$

Also, let

$$
\widetilde{J}(s)=\int \varepsilon(s, y) \int_{y}^{+\infty}\left(\frac{Q}{2}+z Q_{z}\right) .
$$

By the exponential decay on the left $(41), J(s)$ is well-defined for all $s \geq 0$, and it follows from (29) that

$$
J_{s}+\frac{\lambda_{s}}{2 \lambda} J+2 \int \varepsilon Q \leq C \int \varepsilon^{2} e^{-\frac{|y|}{2}} .
$$

By (28), for $\alpha_{0}$ small enough $\left(E_{0} \leq 0\right)$,

$$
2 \int \varepsilon Q \geq 2 \lambda^{2}\left|E_{0}\right|+\frac{1}{2} \int \varepsilon_{y}^{2}-C \int \varepsilon^{2} e^{-|y|}
$$

Therefore,

$$
J_{s}+\frac{\lambda_{s}}{2 \lambda} J+\frac{1}{2} \int \varepsilon_{y}^{2}+2 \lambda^{2}\left|E_{0}\right| \leq C \int \varepsilon^{2} e^{-\frac{|y|}{2}}
$$

Multiplying this relation by $\sqrt{\frac{\lambda(s)}{\lambda\left(s_{1}\right)}}$ we find

$$
\left(\sqrt{\frac{\lambda(s)}{\lambda\left(s_{1}\right)}} J\right)_{s}+\frac{1}{2} \sqrt{\frac{\lambda(s)}{\lambda\left(s_{1}\right)}} \int \varepsilon_{y}^{2}+2 \sqrt{\frac{\lambda(s)}{\lambda\left(s_{1}\right)}} \lambda^{2}\left|E_{0}\right| \leq C \sqrt{\frac{\lambda(s)}{\lambda\left(s_{1}\right)}} \int \varepsilon^{2} e^{-\frac{|y|}{2}} .
$$

Since for $s \in\left[s_{1}, s_{2}\right], 1 \geq \sqrt{\frac{\lambda(s)}{\lambda\left(s_{1}\right)}} \geq \frac{1}{\sqrt{1.1}} \geq \frac{1}{2}$, we obtain

$$
\left(\sqrt{\frac{\lambda(s)}{\lambda\left(s_{1}\right)}} J\right)_{s}+\frac{1}{4} \int \varepsilon_{y}^{2}+\lambda^{2}\left|E_{0}\right| \leq C \int \varepsilon^{2} e^{-\frac{|y|}{2}}
$$

After integration between $s_{1}$ and $s_{2}$, we obtain

$$
\left[\sqrt{\frac{\lambda\left(s_{2}\right)}{\lambda\left(s_{1}\right)}} J\left(s_{2}\right)-J\left(s_{1}\right)\right]+\frac{1}{4} \int_{s_{1}}^{s_{2}} \int \varepsilon_{y}^{2}+\left|E_{0}\right| \int_{s_{1}}^{s_{2}} \lambda^{2} \leq C \int_{s_{1}}^{s_{2}} \int \varepsilon^{2} e^{-\frac{|y|}{2}} .
$$

Next, we have

$$
\sqrt{\frac{\lambda\left(s_{2}\right)}{\lambda\left(s_{1}\right)}} J\left(s_{2}\right)-J\left(s_{1}\right)=\frac{1}{4}\left(1-\sqrt{\frac{1}{1.1}}\right)\left(\int Q\right)^{2}+\sqrt{\frac{1}{1.1}} \widetilde{J}\left(s_{2}\right)-\widetilde{J}\left(s_{1}\right) .
$$

Note that from the exponential control on the left of $\varepsilon(s, y)$, and the decay properties of the functions $Q$ and $Q_{y}$, for all $s \in\left[s_{1}, s_{2}\right]$,

$$
|\widetilde{J}(s)| \leq C \alpha_{0}^{1 / 4}\left|\int_{y}^{+\infty} \frac{Q}{2}+z Q_{z}\right|_{L^{\infty}} \int_{y<0} e^{C_{2} y}+C|\varepsilon(s)|_{L^{\infty}} \int_{y>0} e^{-C y} \leq C \alpha_{0}^{1 / 4} .
$$


Therefore, for $\alpha_{0}$ small enough,

$$
\begin{aligned}
\sqrt{\frac{\lambda\left(s_{2}\right)}{\lambda\left(s_{1}\right)}} J\left(s_{2}\right)-J\left(s_{1}\right) & \geq \frac{1}{4}\left(1-\sqrt{\frac{1}{1.1}}\right)\left(\int Q\right)^{2}-2 C \alpha_{0}^{1 / 4} \\
& \geq \frac{1}{8}\left(1-\sqrt{\frac{1}{1.1}}\right)\left(\int Q\right)^{2} .
\end{aligned}
$$

Thus, there exists $C_{I}>0$ such that

$$
C_{I} \int_{s_{1}}^{s_{2}} \int \varepsilon^{2} e^{-\frac{|y|}{2}} \geq 1+\int_{s_{1}}^{s_{2}} \int \varepsilon_{y}^{2}+\left|E_{0}\right| \int_{s_{1}}^{s_{2}} \lambda^{2} .
$$

Step 3. Proof of (43). By Proposition 2, and the orthogonality conditions imposed on $\varepsilon(s)$ (see Lemma 1),

$$
\left(\int \Psi_{A_{0}} \varepsilon^{2}\right)_{s} \leq-\delta_{0} \int\left(\varepsilon^{2}+\varepsilon_{y}^{2}\right) e^{-\frac{|y|}{A_{0}}}+\frac{1}{\delta_{0}}\left(\int \varepsilon Q\right)^{2} .
$$

Integrating between $s_{1}$ and $s_{2}$, we obtain:

$$
\int \Psi_{A_{0}} \varepsilon^{2}\left(s_{2}\right)-\int \Psi_{A_{0}} \varepsilon^{2}\left(s_{1}\right) \leq-\delta_{0} \int_{s_{1}}^{s_{2}} \int\left(\varepsilon^{2}+\varepsilon_{y}^{2}\right) e^{-\frac{|y|}{A_{0}}}+\frac{1}{\delta_{0}} \int_{s_{1}}^{s_{2}}\left(\int \varepsilon Q\right)^{2} .
$$

On the one hand, we have

$$
\left|\int \Psi_{A_{0}} \varepsilon^{2}\left(s_{2}\right)-\int \Psi_{A_{0}} \varepsilon^{2}\left(s_{1}\right)\right| \leq C A_{0}|\varepsilon(s)|_{L^{2}}^{2} \leq C^{\prime} \alpha_{0},
$$

since $\left|\Psi_{A_{0}}(y)\right| \leq C A_{0}$ and $\int \varepsilon^{2} \leq C \alpha_{0}$.

On the other hand, by $\int \varepsilon^{2} \leq C \alpha_{0}$,

$$
\int_{s_{1}}^{s_{2}}\left(\int \varepsilon Q\right)^{2} \leq \sup _{\left(s_{1}, s_{2}\right)}\left(\int|\varepsilon Q|\right) \int_{s_{1}}^{s_{2}}\left|\int \varepsilon Q\right| \leq C \alpha_{0}^{1 / 2} \int_{s_{1}}^{s_{2}}\left|\int \varepsilon Q\right| .
$$

By (28),

$$
\left|\int \varepsilon Q\right| \leq \lambda^{2}\left|E_{0}\right|+\int \varepsilon_{y}^{2}+C \int \varepsilon^{2} e^{-|y|} .
$$

Therefore,

$$
\begin{aligned}
\int_{s_{1}}^{s_{2}}\left(\int \varepsilon Q\right)^{2} & \leq C \alpha_{0}^{1 / 2} \int_{s_{1}}^{s_{2}}\left(\lambda^{2}\left|E_{0}\right|+\int \varepsilon_{y}^{2}+\int \varepsilon^{2} e^{-|y|}\right) \\
& \leq C \alpha_{0}^{1 / 2} \int_{s_{1}}^{s_{2}}\left(\lambda^{2}\left|E_{0}\right|+\int \varepsilon_{y}^{2}\right)+\frac{\delta_{0}^{2}}{2} \int_{s_{1}}^{s_{2}} \int \varepsilon^{2} e^{-\frac{|y|}{A_{0}}}
\end{aligned}
$$

for $\alpha_{0}$ such that $C \alpha_{0}^{1 / 2} \leq \delta_{0}^{2} / 2$. 
Finally, we obtain, for $\alpha_{0}$ small,

$$
\int_{s_{1}}^{s_{2}} \int\left(\varepsilon^{2}+\varepsilon_{y}^{2}\right) e^{-\frac{|y|}{A_{0}}} \leq C_{I I}\left[\alpha_{0}+\alpha_{0}^{1 / 2} \int_{s_{1}}^{s_{2}}\left(\lambda^{2}\left|E_{0}\right|+\int \varepsilon_{y}^{2}\right)\right]
$$

thus the proof of (43) is complete, and Proposition 5 is proved.

\section{Lower bounds on the blow-up rate and stability of $Q$ as blow-up profile}

In this section, we prove the two main results. First, we show that Theorem 2 follows easily from Theorem 1 . The rest of the section is then devoted to the proof of Theorem 1 .

4.1. Theorem 1 implies Theorem 2. The proof of the lower bounds on the blow-up rate follows directly from the fact that in the rescaled variables, the solution behaves like $Q$, and the fact that the solution $v(t, x)=Q(x-t)$ satisfies $\frac{d \lambda_{v}}{d t}=0$.

Consider a solution $u(t)$ blowing up in a finite time $T>0$, with $\int u^{2}<\int Q^{2}+\alpha_{0}$, where $\alpha_{0}$ is defined as in Theorem 1. From Theorem 1, we have

$$
\varepsilon(s) \rightarrow 0, \quad \text { in } H^{1}(\mathbf{R}), \text { as } s \rightarrow+\infty .
$$

This implies, by the compact embedding from $H^{1}(\mathbf{R})$ to $L_{\text {loc }}^{2}(\mathbf{R})$, that

$$
\varepsilon(s) e^{-\frac{|y|}{\sqrt{2}}} \rightarrow 0, \quad \text { in } L^{2}(\mathbf{R}) \text { strong, as } s \rightarrow+\infty .
$$

From Lemma 2 (iii), we have $\left|\frac{\lambda_{s}}{\lambda}\right| \leq C\left(\int \varepsilon^{2}(s) e^{-\frac{|y|}{2}}\right)^{1 / 2}$, and so $\frac{\lambda_{s}(s)}{\lambda(s)} \rightarrow 0$, as $s \rightarrow+\infty$. Since $\frac{d s}{d t}=1 / \lambda^{3}$, this is equivalent to

$$
\left(\lambda^{3}(t)\right)_{t}=3 \lambda^{2}(t) \lambda_{t}(t) \rightarrow 0, \quad \text { as } t \uparrow T .
$$

By integration, it follows that $\frac{\lambda^{3}(t)}{(T-t)} \rightarrow 0$, as $t \uparrow T$, and then (20) yields Theorem 2.

4.2. Proof of the stability of the blow-up profile. In this subsection, we prove Theorem 1. We consider a solution $u(t)$ of (1) blowing up in finite or infinite time $T>0$. We assume that $\int u^{2}=\int Q^{2}+\alpha_{0}$ where $\alpha_{0}$ is small so that we have the decomposition described in Section 2. Since $\lambda^{1 / 2}(s) u(s, \lambda(s) y+$ $x(s))=Q(y)+\varepsilon(s, y)$, the result is implied by

$$
\varepsilon(s) \rightarrow 0 \quad \text { in } H^{1}(\mathbf{R}), \text { as } s \rightarrow+\infty .
$$

The proof of (44) is divided in two parts. 
In part $\mathrm{A}$, we prove (44) for a subsequence of time $\left(t_{n}\right)$ related to a monotonicity property of the oscillations of $\left|u_{x}(t)\right|_{L^{2}}$ or equivalently $\lambda(t)$, namely for all $t \geq t_{n}, \lambda(t) \leq \lambda\left(t_{n}\right), \lambda\left(t_{n+1}\right)=\frac{\lambda\left(t_{n}\right)}{1.1}$. Under this control, we are able to construct a limit object $\widetilde{u}(t)$ with uniform exponential decay for $y<0$; this property is one of the keys to obtain the contradiction with $\widetilde{u}(t)$ as in Section 3 . However, unlike Section 3, we do not have control of the growth of the gradient $\left|u_{x}(t)\right|_{L^{2}} \leq \frac{C}{(T-t)^{1 / 3}}$. Thus, two possibilities will have to be considered for $\widetilde{u}(t)$ :

(i) Either there exists $t>0$ such that $\tilde{\lambda}(t)=\frac{1}{1.1}$. Thus the solution grows in $H^{1}$ for $t>0$ and then we are reduced to the case of $\S 3.2$;

(ii) Or for all $t>0, \frac{1}{1.1}<\tilde{\lambda}(t) \leq 1$ which means that the solution stays bounded, and the Liouville theorem of [12] giving a classification of such solutions gives a contradiction. More precisely, we recall the following result of asymptotic stability of $Q$.

Proposition 6 (Asymptotic stability of $Q$ [12]). There exists $\alpha_{I I}>0$ such that if a solution $u(t)$ of (1) defined for $t \geq 0$ is such that

(i) $\int u^{2}<\int Q^{2}+\alpha_{I I}$,

(ii) $E(u) \leq 0$,

(iii) for all $t \geq 0, \frac{1}{2} \leq \lambda(t) \leq 1$, then $\varepsilon(t) \rightarrow 0$ in $H^{1}(\mathbf{R})$, as $t \rightarrow+\infty$.

This is a direct consequence of Theorem 2 in [12], and Lemma 1. Note that the result is strongly related to a classification of bounded solutions close to $Q$, characterizing the soliton $Q(x-t)$.

In both (i) and (ii), $L^{1}$ dispersion (Proposition 1) and $L^{2}$ dispersion (Proposition 2) play a crucial role to obtain a contradiction.

In part $\mathrm{B}$, we extend the result to the whole sequence $t \rightarrow T$, using arguments in $L^{2}$. More precisely, we use almost-monotonicity of the mass to the left of the solution when $t$ increases, and to the right of the solution when $t$ decreases (using the argument backwards, and the invariance of equation (1): if $u(t, x)$ is solution then $u(-t,-x)$ is solution). Therefore, the $L^{2}$ mass of $Q+\varepsilon(t)$ on compact sets in space is controlled for all time, and we conclude by using the variational characterization of $Q$.

A. Convergence to $Q$ as $t_{n} \rightarrow T$. We define an increasing sequence $t_{n} \rightarrow T$ such that

$$
\lambda\left(t_{n}\right)=\frac{1}{(1.1)^{n}}, \quad \text { for all } t \in\left[t_{n}, T\right), \lambda(t) \leq \lambda\left(t_{n}\right) .
$$

Such a sequence $\left(t_{n}\right)$ allows us to obtain the property of exponential decay on the left for the limit object. Indeed, such a property is known to hold when there is a control of type (45) on the solution (see $§ 2.3$ ). 
The objective of this subsection is to prove the following proposition.

Proposition 7 (Convergence as $t_{n} \rightarrow T$ ). There exists $\alpha_{I I I}>0$ such that if $\int u^{2} \leq \int Q^{2}+\alpha_{I I I}$, then we have

$$
\varepsilon\left(t_{n}\right) \rightarrow 0 \quad \text { in } H^{1}(\mathbf{R}) \text { weak, as } n \rightarrow+\infty \text {. }
$$

\section{Proof of Proposition 7.}

Step 1. Introduction of the limit dynamics. For $\bar{x} \in \mathbf{R}$ and $\bar{t} \in\left[-\frac{t_{n}}{\lambda^{3}\left(t_{n}\right)}\right.$, $\left.\frac{T-t_{n}}{\lambda^{3}\left(t_{n}\right)}\right)$, we define

$$
u_{n}(\bar{t}, \bar{x})=\lambda^{1 / 2}\left(t_{n}\right) u\left(t_{n}+\lambda^{3}\left(t_{n}\right) \bar{t}, \lambda\left(t_{n}\right) \bar{x}+x\left(t_{n}\right)\right) .
$$

By scaling invariance of (1), the function $u_{n}(\bar{t}, \bar{x})$ is a solution of (1) with blow-up time $T_{n}=\frac{T-t_{n}}{\lambda^{3}\left(t_{n}\right)}\left(T_{n}=+\infty\right.$ if $\left.T=+\infty\right)$. Note that $\frac{T-t_{n}}{\lambda^{3}\left(t_{n}\right)} \rightarrow+\infty$ as $n \rightarrow+\infty$ is possible.

We check easily that the sequence $\left(u_{n}\right)$ satisfies the following properties:

(i) $\int u_{n}^{2}=\int Q^{2}+\alpha_{0}$,

(ii) $\lim _{n \rightarrow+\infty} E\left(u_{n}\right)=0$ (since $E\left(u_{n}\right)=\lambda^{2}\left(t_{n}\right) E(u)$ ),

(iii) $\lambda_{n}(0)=1, x_{n}(0)=0$, for all $\bar{t} \in\left[0, T_{n}\right), \lambda_{n}(\bar{t}) \leq 1$,

(iv) $\left|u_{n}(0)\right|_{H^{1}} \leq C$,

where $\lambda_{n}(\bar{t})$ and $x_{n}(\bar{t})$ are the geometrical parameters associated to $u_{n}$. Note that $\varepsilon\left(t_{n}\right)=\varepsilon_{n}(0)$, where $\varepsilon_{n}$ is associated to $u_{n}$ as in $\S 2.1$.

The proof of the proposition is by contradiction. Assume that up to the extraction of a subsequence (still denoted $\varepsilon_{n}$ ), there exists $\widetilde{\varepsilon}(0) \not \equiv 0, \widetilde{\varepsilon}(0) \in$ $H^{1}(\mathbf{R})$ such that

$$
\varepsilon_{n}(0) \rightarrow \widetilde{\varepsilon}(0) \quad \text { in } H^{1}(\mathbf{R}), \text { as } n \rightarrow+\infty .
$$

We denote by $\widetilde{u}(t)$ the solution of (1) with initial data $\widetilde{u}(0)=Q+\widetilde{\varepsilon}(0)$, and $\widetilde{T}>0$ its maximal time of existence. We have easily the following properties of $\widetilde{u}$.

Lemma 7 (First properties of the limit object $\widetilde{u}$ ). We have

(i) $\int Q^{2} \leq \int \widetilde{u}^{2} \leq \int Q^{2}+\alpha_{0}$,

(ii) $E(\widetilde{u}) \leq 0$,

(iii) $\widetilde{\lambda}(0)=1, \widetilde{x}(0)=0$, for all $t \in[0, \widetilde{T}), \widetilde{\lambda}(t) \leq 1$.

Proof. (i) and (ii) follow from standard variational arguments given in $\S 3.1$. 
(iii) Since $E(\widetilde{u}) \leq 0$, we can consider $\widetilde{\varepsilon}, \widetilde{\lambda}$ and $\widetilde{x}$ associated to the decomposition of $\widetilde{u}$. Since $\int x Q_{x} \varepsilon_{n}(0)=\int x\left(\frac{Q}{2}+x Q_{x}\right) \varepsilon_{n}(0)=0$, by weak convergence, this is still true for $\widetilde{\varepsilon}(0)$. Since $\widetilde{u}(0)=Q+\widetilde{\varepsilon}(0), \widetilde{\lambda}(0)=1$ and $\widetilde{x}(0)=0$.

We recall from Lemma 6 that for any $t_{0} \in\left[0, \min \left(\widetilde{T}, \underline{\lim } T_{n}\right)\right)$,

$$
\begin{aligned}
& \text { for all } t \in\left[0, t_{0}\right], \quad u_{n}(t) \rightarrow \widetilde{u}(t) \quad \text { in } H^{1}(\mathbf{R}), \\
& u_{n} \rightarrow \widetilde{u} \quad \text { in } C\left(\left[0, t_{0}\right], L_{\text {loc }}^{2}(\mathbf{R})\right), \quad \lambda_{n} \rightarrow \widetilde{\lambda} \quad \text { in } C\left(\left[0, t_{0}\right], \mathbf{R}\right) \text { as } n \rightarrow+\infty .
\end{aligned}
$$

We claim that $\widetilde{T} \leq \underline{\lim } T_{n}$. By contradiction, $\widetilde{T}>\underline{\lim } T_{n}$ and the preceding property imply that there exists $c>0$ such that for all $t_{0} \in\left[0, \underline{\lim } T_{n}\right)$,

$$
\exists n\left(t_{0}\right), \text { for all } n \geq n\left(t_{0}\right), \lambda_{n}\left(t_{0}\right) \geq c \text {. }
$$

By (20) it follows that $\left|u_{n x}\left(t_{0}\right)\right|_{L^{2}} \leq C$; thus by the well-posedness of the Cauchy problem in $H^{1}, u_{n}(t)$ is defined on $\left[t_{0}, t_{0}+\tau\right]$, where $\tau>0$ is independent of $t_{0}$, and in particular $\underline{\lim } T_{n} \geq t_{0}+\tau$. This is a contradiction.

Thus, we obtain for all $t \in[0, \widetilde{T}), \widetilde{\lambda}(t) \leq 1$ and Lemma 7 is proved.

Now we define $\tau=\inf \left\{t>0, \tilde{\lambda}(t)=\frac{1}{1.1}\right\}$, possibly $\tau=+\infty$. It follows from the definition of $\tau$ that

$$
\text { for all } t \in[0, \tau), \quad \frac{1}{1.1} \leq \widetilde{\lambda}(t) \leq 1 .
$$

Using crucially the control of $\widetilde{\lambda}(t)$, we have the following lemma, proved in the last step.

Lemma 8 (Exponential decay of $\widetilde{\varepsilon}$ on the left on $[0, \tau)$ ). There exists $C>0$ such that for $\alpha_{0}$ small enough,

$$
\text { for all } y<0, \text { for all } t \in[0, \tau), \quad|\widetilde{\varepsilon}(t, y)| \leq C \alpha_{0}^{1 / 4} e^{-\frac{|y|}{28}} .
$$

Step 2. The main argument. The idea is to use two crucial dynamical arguments which say that a solution different from $Q$ has to disperse some mass on the left. Therefore, we have a result of nonexistence of $\widetilde{u}$, which is a contradiction. Consider two cases.

(a) Focusing regime under exponential decay on the left: $\tau<+\infty$. The contradiction follows from Proposition 5, for $\alpha_{0}$ small. Indeed, from Lemma 7 and Lemma 8 , we obtain a solution $\widetilde{u}(t)$ satisfying

(i) $\int \widetilde{u}^{2}(t) \leq \int Q^{2}+\alpha_{0}$,

(ii) $E(\widetilde{u}) \leq 0$, 
(iii) $\widetilde{\lambda}(0)=1$ and for all $t \in[0, \widetilde{T}), \widetilde{\lambda}(t) \leq 1 ; \quad \widetilde{\lambda}(\tau)=\frac{1}{1.1}$, for all $t \in$ $[0, \tau), \frac{1}{1.1} \leq \widetilde{\lambda}(t) \leq 1$,

(iv) for all $y<0$, for all $t \in[0, \tau), \quad|\widetilde{\varepsilon}(t, y)| \leq C \alpha_{0}^{1 / 4} e^{-\frac{|y|}{28}}$.

(b) Regular regime under $L^{2}$ compactness of the solution: $\tau=+\infty$. We have a solution $\widetilde{u}(t)$, defined for $t \geq 0$ such that

$$
\text { for all } t \geq 0, \quad \frac{1}{1.1} \leq \widetilde{\lambda}(t) \leq 1 \text {. }
$$

Using Proposition 6 and the compact embedding of $H^{1}(\mathbf{R})$ in $L_{\text {loc }}^{2}(\mathbf{R})$, we have first:

$$
\widetilde{\varepsilon}(t) \rightarrow 0 \text { in } L_{\text {loc }}^{2}(\mathbf{R}) \text {, as } t \rightarrow+\infty .
$$

Now, using the exponential decay and a property of equation (1), we have

Claim. For all $\delta>0$, there exist $R(\delta)>0$, for all $t \geq 0$,

$$
\int_{|y|>R(\delta)} \widetilde{\varepsilon}^{2}(t, y) d y \leq \delta .
$$

Proof. On the one hand, by the uniform exponential decay of $\widetilde{\varepsilon}$ on the left given by Lemma 8 , we have the $L^{2}$ compactness of $\widetilde{\varepsilon}(s)$ on the left (i.e. for $y<0)$.

On the other hand, it follows by using backwards the almost monotonicity of the mass that $\widetilde{\varepsilon}$ is $L^{2}$ compact on the right. Indeed, assume for the sake of contradiction that there exists $\delta>0$ and a sequence $\widetilde{t}_{n}$ be such that $\int_{y>n} \widetilde{\varepsilon}^{2}\left(\widetilde{t}_{n}, y\right) d y \geq \delta$. Since $t \mapsto \widetilde{\varepsilon}(t)$ is continuous in $L^{2}$, we necessarily have $\widetilde{t}_{n} \rightarrow+\infty$. Let $x_{0}$ be such that $C_{0} e^{-\frac{x_{0}}{3}}<\delta / 2$. Since, by the invariance of (1), $\widetilde{u}\left(\widetilde{t}_{n}-\bar{t},-x\right)$ is a solution, for $\bar{t} \in\left[0, \widetilde{t}_{n}\right]$, by using Lemma 5 , we have $\mathcal{I}_{x_{0}}\left(\widetilde{t}_{n}\right) \geq \mathcal{I}_{x_{0}}(0)-C_{0} e^{-x_{0} / 3}$. Thus $\mathcal{I}_{x_{0}}\left(\widetilde{t}_{n}\right) \geq \delta-\delta / 2=\delta / 2$, and for $n$ large we obtain $\int_{y>n} \widetilde{\varepsilon}^{2}(0, y) d y \geq \frac{\delta}{4}$. But this is a contradiction for $n$ large. Thus the claim is proved.

In conclusion, $\widetilde{\varepsilon}(t) \rightarrow 0$ in $L^{2}(\mathbf{R})$ as $t \rightarrow+\infty$, and passing to the limit as $t \rightarrow+\infty$ in the mass conservation $\int \widetilde{u}^{2}(0)=\int \widetilde{u}^{2}(t)=\int Q^{2}+2 \int \widetilde{\varepsilon}(t) Q+\int \widetilde{\varepsilon}^{2}(t)$, we obtain

$$
\int \widetilde{u}^{2}(0)=\int Q^{2}
$$

Since $E(\widetilde{u}(0)) \leq 0$, by the characterization of $Q(5)$, there exist $\lambda_{0}>0$ and $x_{0} \in \mathbf{R}$ such that $\widetilde{u}(0)=Q+\widetilde{\varepsilon}(0)=\lambda_{0}^{1 / 2} Q\left(\lambda_{0}\left(x+x_{0}\right)\right)$. Since $\left|\lambda_{0}-1\right|+\left|x_{0}\right| \leq$ $C \sqrt{\alpha_{0}}$ (from $\left.|\widetilde{\varepsilon}(0)|_{L^{2}} \leq C \sqrt{\alpha_{0}}\right)$, we obtain by the orthogonality conditions on $\widetilde{\varepsilon}(0)$ that $\lambda_{0}=1$ and $x_{0}=0$; thus $\widetilde{\varepsilon}(0) \equiv 0$. This is a contradiction. 
Therefore, we have a contradiction in cases (a), (b), and the proposition is proved.

Step 3. Exponential decay of $\widetilde{\varepsilon}$ on the left on a "doubling" interval of time. This step is devoted to the proof of Lemma 8. We claim that Lemma 8 is a consequence of

$\exists C_{1}>0$, for all $x_{0}<0$, for all $t \in[0, \tau), \int_{2 x_{0}<x<x_{0}} \widetilde{u}^{2}(t, x+\widetilde{x}(t)) d x \leq C_{1} e^{-\frac{\left|x_{0}\right|}{12}}$.

Indeed, by summation of (47), properties of $Q$ and control of $\widetilde{\lambda}(t)$ on $[0, \tau)$, we have directly

$$
\text { for all } y_{0}<0 \text {, for all } t \in[0, \tau), \quad \int_{y<y_{0}} \widetilde{\varepsilon}^{2}(t, y) d y \leq C e^{-\frac{\left|y_{0}\right|}{14}},
$$

and we obtain Lemma 8 as in the proof of Corollary 1.

Now, we prove the exponential decay on $\widetilde{u}(47)$. As in the proof of the exponential decay in $\S 3.1$, it follows from monotonicity properties on $u_{n}$ in $L_{\text {loc }}^{2}$ and recurrence of $\widetilde{u}$ on $u_{n}$. Here, we do not assume $\left|u_{x}(t)\right|_{L^{2}} \leq \frac{C}{(T-t)^{1 / 3}}$, and thus $\widetilde{u}$ does not concentrate as a Dirac mass in $L^{2}$ at the blow-up time. However, considering a quantity $\mathcal{J}$ which measures the mass lost on the left as $n \rightarrow+\infty$, we still have similar estimates as in $\S 3.1$.

We prove (47) by contradiction. Let $a_{0}$ and $C_{0}$ be defined as in Lemma 5 . Let $a_{1}>a_{0}$ and $C_{1}>0$ be chosen later. It is sufficient to prove (47) for $x_{0}<-a_{1}$. Assume that there exists $t_{0} \in[0, \tau)$, and $x_{0}<-a_{1}$ such that

$$
\int_{2 x_{0}<x<x_{0}} \widetilde{u}^{2}\left(t_{0}, x+\widetilde{x}\left(t_{0}\right)\right) d x \geq C_{1} e^{-\frac{\left|x_{0}\right|}{12}} .
$$

Since $u_{n}\left(t_{0}, x+x_{n}\left(t_{0}\right)\right) \rightarrow \widetilde{u}\left(t_{0}, x+\widetilde{x}\left(t_{0}\right)\right)$ in $L_{\text {loc }}^{2}(\mathbf{R})$, there exists $n_{0}$ such that

$$
\text { for all } n \geq n_{0}, \quad \int_{2 x_{0}<x<x_{0}} u_{n}^{2}\left(t_{0}, x+x_{n}\left(t_{0}\right)\right) d x \geq \frac{C_{1}}{2} e^{-\frac{\left|x_{0}\right|}{12}} .
$$

For the same choice of function $\psi$ as in section 2.3 , for $x_{1} \in \mathbf{R}$, we define for $t \in\left[t_{0}, T_{n}\right)$,

$$
\mathcal{J}_{x_{1}, n}(t)=\int u_{n}^{2}(t, x)\left(1-\psi\left(x-x_{n}\left(t_{0}\right)-x_{1}-\frac{1}{4}\left(x_{n}(t)-x_{n}\left(t_{0}\right)\right)\right)\right) d x .
$$

Note that this quantity measures the mass on the left since $\psi(x) \rightarrow 0$ as $x \rightarrow-\infty$, and $\psi(x) \rightarrow 1$ as $x \rightarrow+\infty$. We claim the following lemma, proved in Appendix B.

Lemma 9 (Limit of $\mathcal{J}_{x_{1}, n}(t)$ ). There exists $\mathcal{J}$ such that the following property holds: $\exists C>0$, for all $\delta_{1}>0$, for all $x_{1}<-a_{1}, \exists n_{1}=n_{1}\left(\delta_{1}, x_{1}\right)$ such that

$$
\text { for all } n \geq n_{1}, \text { for all } t \in\left[t_{0}, T_{n}\right), \quad\left|\mathcal{J}_{x_{1}, n}(t)-\mathcal{J}\right| \leq \delta_{1}+C e^{\frac{x_{1}}{8}}
$$


Remark. In fact, $\mathcal{J}$ measures the mass lost at the blow-up time at the left of the soliton in the rescaled variable.

This result provides a contradiction and thus proves (47). Indeed, on the one hand, for $n \geq n_{0}$, we have

$$
\mathcal{J}_{x_{0}, n}\left(t_{0}\right)-\mathcal{J}_{2 x_{0}, n}\left(t_{0}\right)=\int u_{n}^{2}\left(t_{0}, x+x_{n}\left(t_{0}\right)\right)\left(\psi\left(x-2 x_{0}\right)-\psi\left(x-x_{0}\right)\right) d x
$$

Since the function $\psi$ is increasing, and since for $\left|x_{0}\right|$ large enough,

$$
\min _{\left(2 x_{0}, x_{0}\right)}\left(\psi\left(x-2 x_{0}\right)-\psi\left(x-x_{0}\right)\right) \geq \frac{1}{4},
$$

we obtain, by taking $a_{1}$ large enough and using (48),

$$
\mathcal{J}_{x_{0}, n}\left(t_{0}\right)-\mathcal{J}_{2 x_{0}, n}\left(t_{0}\right) \geq \frac{1}{4} \int_{2 x_{0}<x<x_{0}} u_{n}^{2}\left(t_{0}, x+x_{n}(t)\right) d x \geq \frac{C_{1}}{8} e^{-\frac{\left|x_{0}\right|}{12}} .
$$

On the other hand, by Lemma 9, applied for $\delta_{1}=\frac{C_{1}}{32} e^{\frac{x_{0}}{12}}$, there exists $n_{1}=n_{1}\left(x_{0}\right)$ such that for all $n \geq n_{1}$,

$$
\mathcal{J}-\frac{C_{1}}{32} e^{\frac{x_{0}}{12}}-C e^{\frac{2 x_{0}}{8}} \leq \mathcal{J}_{2 x_{0}, n}\left(t_{0}\right) \quad \text { and } \quad \mathcal{J}_{x_{0}, n}\left(t_{0}\right) \leq \mathcal{J}+\frac{C_{1}}{32} e^{\frac{x_{0}}{12}}+C e^{\frac{x_{0}}{8}} .
$$

Therefore, $\mathcal{J}_{x_{0}, n}\left(t_{0}\right)-\mathcal{J}_{2 x_{0}, n}\left(t_{0}\right) \leq \frac{C_{1}}{16} e^{\frac{x_{0}}{12}}+2 C e^{\frac{x_{0}}{8}}$. Thus, we obtain a contradiction by taking $C_{1}>32 C$ (recall that $C$ here is independent of $x_{0}$ ) for $n>n_{0}, n_{1}$.

B. Convergence to $Q$ for $t \rightarrow T$. Note that for $\left(t_{n}\right)$ defined in part A, $t_{1}<t_{2}<\ldots<t_{n}<T$, and $t_{n} \rightarrow T$. We now claim the following proposition, which completes the proof of Theorem 1 .

Proposition 8 (Convergence for $t \rightarrow T$ ). There exists $\alpha_{I V}>0$ such that if $\int u^{2} \leq \int Q^{2}+\alpha_{I V}$, then

$$
\varepsilon(t) \rightarrow 0 \quad \text { in } H^{1}(\mathbf{R}) \text {, as } t \uparrow T .
$$

Proof. We argue by contradiction. Suppose that there exists $\widetilde{\varepsilon} \in H^{1}(\mathbf{R})$, $\widetilde{\varepsilon} \not \equiv 0$, and a subsequence of $\left(t_{n}\right)$ denoted $\left(t_{n^{\prime}}\right)$, such that for all $n^{\prime}$ there is $t_{n^{\prime}}^{1} \in\left[t_{n^{\prime}}, t_{n^{\prime}+1}\right]$, such that

$$
\varepsilon\left(t_{n^{\prime}}^{1}\right) \rightarrow \widetilde{\varepsilon} \text { in } H^{1}(\mathbf{R}) \text { as } n^{\prime} \rightarrow+\infty .
$$

First, we use again the fact that $u_{n}$ is recurrent in $u$ and the mass properties (Lemma 9) to obtain that the $L^{2}$ mass of $u\left(t_{n}\right)$ as $n \rightarrow+\infty$ has a limit at the left $\left(x<x\left(t_{n}\right)\right)$ and at the right $\left(x>x\left(t_{n}\right)\right)$.

Then, we use the almost-monotonicity property twice to extend this result to $u\left(t_{n^{\prime}}^{1}\right)$ as $n^{\prime} \rightarrow+\infty$ : 
(i) the first time on $u(t, x)$, from $t_{n^{\prime}}$ to $t_{n^{\prime}}^{1}$ for $x<0$.

(ii) the second time on $u(-t,-x)$, which is also a solution. $L^{2}$ estimates at $t_{n^{\prime}+1}$ give $L^{2}$ estimates at $t_{n^{\prime}}^{1}$ for $x>0$. Here we use crucially $\lambda\left(t_{n^{\prime}+1}\right)=\frac{\lambda\left(t_{n^{\prime}}\right)}{1.1}$, to be able to use the almost-monotonicity property on the interval $\left[t_{n^{\prime}}, t_{n^{\prime}+1}\right]$.

We then conclude by energy arguments (variational characterization of $Q$; see $(5))$.

Step 1. Limit of the mass of $\varepsilon\left(t_{n}\right)$ as $n \rightarrow+\infty$. From Lemma 9 and Proposition 7, for $\mathcal{J}$ defined as in Lemma 9, we claim that for all $y_{1} \in \mathbf{R}$,

$$
\lim _{n \rightarrow+\infty} \int_{y<y_{1}} \varepsilon^{2}\left(t_{n}, y\right) d y=\mathcal{J} \text { and } \lim _{n \rightarrow+\infty} \int_{y>y_{1}} \varepsilon^{2}\left(t_{n}, y\right) d y=\alpha_{0}-\mathcal{J} .
$$

Proof of (50). From the fact that $\varepsilon\left(t_{n}\right) \rightarrow 0$ in $H^{1}(\mathbf{R})$, by classical compactness arguments, we have $\varepsilon\left(t_{n}\right) \rightarrow 0$ in $L_{\text {loc }}^{2}$ strong. It follows that $\overline{\mathcal{J}}=\varlimsup_{n \rightarrow+\infty} \int_{y<y_{1}} \varepsilon^{2}\left(t_{n}, y\right) d y$ and $\underline{\mathcal{J}}=\underline{\lim }_{n \rightarrow+\infty} \int_{y<y_{1}} \varepsilon^{2}\left(t_{n}, y\right) d y$ are both independent of $y_{1}$.

Now, consider $u_{n}(t, x)=\lambda^{1 / 2}\left(t_{n}\right) u\left(t_{n}+\lambda^{3}\left(t_{n}\right) t, \lambda\left(t_{n}\right) x+x\left(t_{n}\right)\right)$, and for $t \in\left[0, T_{n}\right)$,

$$
\mathcal{J}_{y_{1}, n}(t)=\int u_{n}^{2}(t, x)\left(1-\psi\left(x-y_{1}-\frac{1}{4} x_{n}(t)\right) d x .\right.
$$

From Lemma 9, applied with $t=0$, since $u_{n}(0)=Q+\varepsilon\left(t_{n}\right)$, we have: for all $\delta_{1}>0$, for all $y_{1}<-a_{1}, \exists n_{1}=n_{1}\left(\delta_{1}, y_{1}\right)$, such that for all $n \geq n_{1}$,

$$
\left|\int(Q+\varepsilon)^{2}\left(t_{n}, y\right)\left(1-\psi\left(y-y_{1}\right)\right) d y-\mathcal{J}\right| \leq \delta_{1}+C e^{\frac{y_{1}}{8}} \text {. }
$$

Thus, $\int_{y<2 y_{1}} \varepsilon^{2}\left(t_{n}, y\right) d y \leq \mathcal{J}+\delta_{1}+C^{\prime} e^{\frac{y_{1}}{8}}$. Therefore, for all $\delta_{1}>0$, for all $y_{1}<-a_{1}$,

$$
\overline{\mathcal{J}}=\varlimsup_{n \rightarrow+\infty} \int_{y<2 y_{1}} \varepsilon^{2}\left(t_{n}, y\right) d y \leq \mathcal{J}+\delta_{1}+C^{\prime} e^{\frac{y_{1}}{8}} .
$$

By $\delta_{1} \rightarrow 0$, we obtain $\overline{\mathcal{J}} \leq \mathcal{J}+C^{\prime} e^{\frac{y_{1}}{8}}$, and then by $y_{1} \rightarrow-\infty$, we have $\overline{\mathcal{J}} \leq \mathcal{J}$. Similarly, using (51), we have $\underline{\mathcal{J}} \geq \mathcal{J}$.

By mass conservation applied at $t=t_{n}$, for all $y_{1} \in \mathbf{R}$,

$$
\int_{y>y_{1}} \varepsilon^{2}\left(t_{n}\right)=\alpha_{0}-2 \int \varepsilon\left(t_{n}\right) Q-\int_{y<y_{1}} \varepsilon^{2}\left(t_{n}\right) .
$$

Therefore, by the fact that $\varepsilon\left(t_{n}\right) \rightarrow 0$ in $L^{2}$ weak, $\lim _{n \rightarrow+\infty} \int_{y>y_{1}} \varepsilon^{2}\left(t_{n}\right)=$ $\alpha_{0}-\mathcal{J}$, and claim (50) is proved.

Step 2. Mass limit of $\varepsilon\left(t_{n^{\prime}}^{1}\right)$ as $n^{\prime} \rightarrow+\infty$. From the property of almostmonotonicity of the $L^{2}$ mass on $u_{n^{\prime}}$, we claim that there exist $a_{n^{\prime}} \rightarrow+\infty$, and $\delta_{n^{\prime}} \rightarrow 0$ such that

$$
\int_{|y|<a_{n^{\prime}}}\left(Q+\varepsilon\left(t_{n^{\prime}}^{1}\right)\right)^{2} \leq \int Q^{2}+\delta_{n^{\prime}}
$$


Proof of (52). Define $\mathcal{I}_{y_{2}, n^{\prime}}(t)=\int u_{n^{\prime}+1}^{2}(-t, x)\left(1-\psi\left(-x+y_{2}+\frac{1}{4} x_{n^{\prime}+1}(-t)\right)\right) d x$. From step 1 , there exist $y_{1, n^{\prime}} \rightarrow-\infty, y_{2, n^{\prime}} \rightarrow+\infty$ and $\delta_{1, n^{\prime}} \rightarrow 0, \delta_{2, n^{\prime}} \rightarrow 0$, such that

$$
\mathcal{J}_{y_{1, n^{\prime}, n^{\prime}}}(0) \geq \mathcal{J}-\delta_{1, n^{\prime}}, \quad \text { and } \quad \mathcal{I}_{y_{2, n^{\prime}}, n^{\prime}}(0) \geq\left(\alpha_{0}-\mathcal{J}\right)-\delta_{2, n^{\prime}} .
$$

By the almost-monotonicity of the $L^{2}$ mass applied on $u_{n^{\prime}}$ (see Lemma 5), we have, for $t_{n^{\prime}}^{2}=\frac{1}{\lambda^{3}\left(t_{n^{\prime}}\right)}\left(t_{n^{\prime}}^{1}-t_{n^{\prime}}\right)$,

$$
\mathcal{J}_{y_{1, n^{\prime}}, n^{\prime}}\left(t_{n^{\prime}}^{2}\right) \geq \mathcal{J}-\delta_{1, n^{\prime}}-C e^{\frac{y_{1, n^{\prime}}}{3}} .
$$

Therefore, by (45),

$\int_{y \leq y_{1, n^{\prime}}}(Q+\varepsilon)^{2}\left(t_{n^{\prime}}^{1}, y\right) d y \geq \int_{y \leq \frac{\lambda\left(t_{n^{\prime}}\right)}{\lambda\left(t_{n^{\prime}}^{1}\right)} y_{1, n^{\prime}}}(Q+\varepsilon)^{2}\left(t_{n^{\prime}}^{1}, y\right) d y \geq \mathcal{J}-\delta_{1, n^{\prime}}-C e^{\frac{y_{1, n^{\prime}}}{3}}$.

Similarly, we use $\mathcal{I}_{y_{2, n^{\prime}}, n^{\prime}}(t)$, the monotonicity property backwards on $u_{n^{\prime}+1}$ (in fact, using that $u_{n^{\prime}+1}(-t,-x)$ is a solution of $(1)$ ), and the fact that by (45)

$$
\lambda\left(t_{n^{\prime}}^{1}\right) \leq \lambda\left(t_{n^{\prime}}\right)=(1.1) \lambda\left(t_{n^{\prime}+1}\right) .
$$

We have $\mathcal{I}_{y_{2, n^{\prime}}, n^{\prime}}(0) \geq\left(\alpha_{0}-\mathcal{J}\right)-\delta_{2, n^{\prime}}$, and so by Lemma 5 , for $t_{n^{\prime}}^{3}=$ $\frac{1}{\lambda^{3}\left(t_{n^{\prime}+1}\right)}\left(t_{n^{\prime}+1}-t_{n^{\prime}}^{1}\right)$,

$$
\mathcal{I}_{y_{2, n^{\prime}, n^{\prime}}}\left(t_{n^{\prime}}^{3}\right) \geq\left(\alpha_{0}-\mathcal{J}\right)-\delta_{2, n^{\prime}}-C e^{-\frac{y_{2, n^{\prime}}}{3}} .
$$

Therefore,

$$
\begin{aligned}
\int_{y \geq \frac{y_{2, n^{\prime}}}{1.1}}(Q+\varepsilon)^{2}\left(t_{n^{\prime}}^{1}, y\right) d y & \geq \int_{y \geq \frac{\lambda\left(t_{n^{\prime}+1}\right)}{\lambda\left(t_{n^{\prime}}\right)} y_{2, n^{\prime}}}(Q+\varepsilon)^{2}\left(t_{n^{\prime}}^{1}, y\right) d y \\
& \geq\left(\alpha_{0}-\mathcal{J}\right)-\delta_{2, n^{\prime}}-C e^{-\frac{y_{2, n^{\prime}}}{3}} .
\end{aligned}
$$

Now, by conservation of mass, and preceding estimates:

$$
\begin{aligned}
\int_{\frac{y_{1, n^{\prime}}}{2} \leq y \leq \frac{y_{2, n^{\prime}}}{2}\left(Q+\varepsilon\left(t_{n^{\prime}}^{1}\right)\right)^{2}=} & \int u_{0}^{2}-\int_{y \leq \frac{y_{1, n^{\prime}}}{2}}\left(Q+\varepsilon\left(t_{n^{\prime}}^{1}\right)\right)^{2} \\
& -\int_{y \geq \frac{y_{2, n^{\prime}}}{2}}\left(Q+\varepsilon\left(t_{n^{\prime}}^{1}\right)\right)^{2} \\
\leq & \int u_{0}^{2}-\mathcal{J}-\left(\alpha_{0}-\mathcal{J}\right)+\delta_{n^{\prime}}=\int Q^{2}+\delta_{n^{\prime}},
\end{aligned}
$$

where $\delta_{n^{\prime}} \rightarrow 0$ as $n^{\prime} \rightarrow+\infty$. Thus the claim is proved.

Step 3. Conclusion. It follows from mass and energy properties of $\varepsilon\left(t_{n^{\prime}}^{1}\right)$. We have by (52): for all $a>0$,

$$
\varlimsup_{n^{\prime} \rightarrow+\infty} \int_{|y|<a}\left(Q+\varepsilon\left(t_{n^{\prime}}^{1}\right)\right)^{2} \leq \int Q^{2} \quad \text { and so } \quad \int(Q+\widetilde{\varepsilon})^{2} \leq \int Q^{2} .
$$


Therefore, since $\varepsilon\left(t_{n^{\prime}}^{1}\right) \rightarrow \widetilde{\varepsilon}$ in $H^{1}$ weak, and by energy arguments as in $\S 3.1$, we have the following properties of $\widetilde{\varepsilon}$ :

$$
|\widetilde{\varepsilon}|_{H^{1}} \leq C \sqrt{\alpha_{0}}, \quad \int y Q_{y} \widetilde{\varepsilon}=\int y\left(\frac{Q}{2}+y Q_{y}\right) \widetilde{\varepsilon}=0, \quad E(Q+\widetilde{\varepsilon}) \leq 0 .
$$

Thus by the variational characterization of $Q$ (see (5)), there exist $\lambda_{0}>0$ and $x_{0} \in \mathbf{R}$ such that $Q+\widetilde{\varepsilon}=\lambda_{0}^{1 / 2} Q\left(\lambda_{0} x+x_{0}\right)$. Then $\left|1-\lambda_{0}\right|+\left|x_{0}\right| \leq C \sqrt{\alpha_{0}}$ follows from the smallness of $\widetilde{\varepsilon}$. From the orthogonality conditions on $\widetilde{\varepsilon}$, we have $\lambda_{0}=1$ and $x_{0}=0$, so that $\widetilde{\varepsilon}=0$, which is a contradiction. This concludes the proof of Proposition 8.

Therefore Theorem 1 is proved.

\section{Appendix A}

The objective is to prove the following result (the function $\Psi_{A}$ is defined in $\S 2.3)$.

Proposition 9 (Local Virial relation). There exist $A_{0}>2, \alpha_{5}>0$ and $\delta_{0}>0$ such that for $\alpha_{0}<\alpha_{5}$

$$
\text { if for all } s \geq 0, \quad \int y Q_{y} \varepsilon(s)=\int y\left(\frac{Q}{2}+y Q_{y}\right) \varepsilon(s)=0, \quad \text { then }
$$

$$
\left(\int \Psi_{A_{0}} \varepsilon^{2}\right)_{s} \leq-\delta_{0} \int\left(\varepsilon^{2}+\varepsilon_{y}^{2}\right) e^{-\frac{|y|}{A_{0}}}+\frac{1}{\delta_{0}}\left(\int \varepsilon Q\right)^{2} .
$$

Proof. Recall the equation satisfied by $\varepsilon(s)$ :

$$
\begin{aligned}
\varepsilon_{s}= & (L \varepsilon)_{y}+\frac{\lambda_{s}}{\lambda}\left(\frac{Q}{2}+y Q_{y}\right)+\left(\frac{x_{s}}{\lambda}-1\right) Q_{y}+\frac{\lambda_{s}}{\lambda}\left(\frac{\varepsilon}{2}+y \varepsilon_{y}\right) \\
& +\left(\frac{x_{s}}{\lambda}-1\right) \varepsilon_{y}-\left(10 Q^{3} \varepsilon^{2}+10 Q^{2} \varepsilon^{3}+5 Q \varepsilon^{4}+\varepsilon^{5}\right)_{y},
\end{aligned}
$$

where $L \varepsilon=-\varepsilon_{x x}+\varepsilon-5 Q^{4} \varepsilon$. As in [11], proof of Lemma 5, regularization arguments allow us to have the following relation, multiplying the equation by $\Psi_{A} \varepsilon$ :

$$
\begin{aligned}
\frac{1}{2}\left(\int \Psi_{A} \varepsilon^{2}\right)_{s}= & -\int L \varepsilon\left(\Psi_{A} \varepsilon\right)_{y} \\
& +\frac{\lambda_{s}}{\lambda} \int\left(\frac{Q}{2}+y Q_{y}\right) \Psi_{A} \varepsilon+\left(\frac{x_{s}}{\lambda}-1\right) \int Q_{y} \Psi_{A} \varepsilon \\
& +\frac{\lambda_{s}}{\lambda} \int\left(\frac{\varepsilon}{2}+y \varepsilon_{y}\right) \Psi_{A} \varepsilon+\left(\frac{x_{s}}{\lambda}-1\right) \int \varepsilon_{y} \Psi_{A} \varepsilon \\
& +\int\left(10 Q^{3} \varepsilon^{2}+10 Q^{2} \varepsilon^{3}+5 Q \varepsilon^{4}\right)\left(\Psi_{A} \varepsilon\right)_{y}-5 \int \Psi_{A} \varepsilon^{5} \varepsilon_{y} .
\end{aligned}
$$


Recall that $\Psi_{A}^{\prime}=\Phi_{A}$ and

$$
\begin{aligned}
& \text { - } \int L \varepsilon\left(\Psi_{A} \varepsilon\right)_{y}=\frac{3}{2} \int \varepsilon_{y}^{2} \Phi_{A}+\frac{1}{2} \int \varepsilon^{2} \Phi_{A} \\
& -\frac{1}{2} \int \varepsilon^{2} \Phi_{A}^{\prime \prime}-\frac{5}{2} \int Q^{4} \Phi_{A} \varepsilon^{2}+10 \int Q_{y} Q^{3} \Psi_{A} \varepsilon^{2}, \\
& \text { - } \int\left(\frac{\varepsilon}{2}+y \varepsilon_{y}\right) \Psi_{A} \varepsilon=-\frac{1}{2} \int y \Phi_{A} \varepsilon^{2}, \\
& \text { - } \int \varepsilon_{y} \Psi_{A} \varepsilon=-\frac{1}{2} \int \Phi_{A} \varepsilon^{2}, \\
& \text { - } \int Q^{i} \varepsilon^{5-i}\left(\Psi_{A} \varepsilon\right)_{y}=\left(\frac{5-i}{6-i}\right) \int Q^{i} \Phi_{A} \varepsilon^{6-i} \\
& -\frac{i}{6-i} \int Q_{y} Q^{i-1} \Psi_{A} \varepsilon^{6-i} \quad \text { for } i=0,1,2,3 .
\end{aligned}
$$

By analogy with the notation

$$
H_{\infty}(\varepsilon, \varepsilon)=\frac{3}{2} \int \varepsilon_{y}^{2}+\frac{1}{2} \int \varepsilon^{2}-\frac{5}{2} \int Q^{4} \varepsilon^{2}+10 \int y Q_{y} Q^{3} \varepsilon^{2},
$$

we set

$$
H_{A}(\varepsilon, \varepsilon)=\frac{3}{2} \int \varepsilon_{y}^{2} \Phi_{A}+\frac{1}{2} \int \varepsilon^{2} \Phi_{A}-\frac{5}{2} \int Q^{4} \Phi_{A} \varepsilon^{2}+10 \int Q_{y} Q^{3} \Psi_{A} \varepsilon^{2} .
$$

We obtain

$$
\begin{aligned}
\frac{1}{2}\left(\int \Psi_{A} \varepsilon^{2}\right)_{s}= & -H_{A}(\varepsilon, \varepsilon)+\frac{\lambda_{s}}{\lambda} \int\left(\frac{Q}{2}+y Q_{y}\right) \Psi_{A} \varepsilon \\
& +\left(\frac{x_{s}}{\lambda}-1\right) \int Q_{y} \Psi_{A} \varepsilon+\frac{1}{2} \int \varepsilon^{2} \Phi_{A}^{\prime \prime}-\frac{1}{2} \frac{\lambda_{s}}{\lambda} \int y \Phi_{A} \varepsilon^{2} \\
& -\frac{1}{2}\left(\frac{x_{s}}{\lambda}-1\right) \int \Phi_{A} \varepsilon^{2}+K_{A}(\varepsilon)+\frac{5}{6} \int \Phi_{A} \varepsilon^{6}
\end{aligned}
$$

where

$$
\begin{aligned}
K_{A}(\varepsilon)= & \frac{20}{3} \int Q^{3} \Phi_{A} \varepsilon^{3}-10 \int Q_{y} Q^{2} \Psi_{A} \varepsilon^{3} \\
& +\frac{15}{2} \int Q^{2} \Phi_{A} \varepsilon^{4}-5 \int Q_{y} Q \Psi_{A} \varepsilon^{4}+4 \int Q \Phi_{A} \varepsilon^{5}-\int Q_{y} \Psi_{A} \varepsilon^{5} .
\end{aligned}
$$

The rest of the proof proceeds in four steps. In the first step, we show that under some perturbation of the orthogonality conditions, $H_{\infty}(\varepsilon, \varepsilon)$ is still positive. In step 2 , we use this result to show that under the orthogonality conditions $\int Q \varepsilon=\int y\left(\frac{Q}{2}+y Q_{y}\right) \varepsilon=0$, for large $A$ (independent of $\left.\alpha_{0}\right), H_{A}(\varepsilon, \varepsilon)$ is positive definite. In step 3 , we remove the condition $\int Q \varepsilon=0$ and obtain an additional term in the inequality. In step 4 , we treat all remaining terms in the expression of $\left(\int \Psi_{A} \varepsilon^{2}\right)_{s}$ to finish the proof of the proposition. 
Step 1. Perturbation of the orthogonality conditions in the limit case. Recall from [12] Part B, that there exists $\delta_{1}>0$ such that

$$
\text { if } \int Q \varepsilon=\int y\left(\frac{Q}{2}+y Q_{y}\right) \varepsilon=0 \quad \text { then } \quad H_{\infty}(\varepsilon, \varepsilon) \geq \delta_{1} \int\left(\varepsilon_{y}^{2}+\varepsilon^{2}\right) \text {. }
$$

We claim that there exists $\delta_{2}>0$ such that

$$
\text { if }|(\varepsilon, Q)|+\left|\left(\varepsilon, y\left(\frac{Q}{2}+y Q_{y}\right)\right)\right| \leq \delta_{2}|\varepsilon|_{H^{1}} \text {, then } H_{\infty}(\varepsilon, \varepsilon) \geq \frac{\delta_{1}}{4} \int\left(\varepsilon_{y}^{2}+\varepsilon^{2}\right) \text {. }
$$

Indeed, take $\varepsilon$ satisfying the assumption of (55). Then

$$
\varepsilon=\varepsilon_{1}+a Q+b y\left(\frac{Q}{2}+y Q_{y}\right)=\varepsilon_{1}+\varepsilon_{2},
$$

where $\left(\varepsilon_{1}, Q\right)=\left(\varepsilon_{1}, y\left(\frac{Q}{2}+y Q_{y}\right)\right)=\left(\varepsilon_{1}, \varepsilon_{2}\right)=0$,

$$
a=\frac{(\varepsilon, Q)}{(Q, Q)}, \quad b=\frac{\left(\varepsilon, y\left(\frac{Q}{2}+y Q_{y}\right)\right)}{\left(y\left(\frac{Q}{2}+y Q_{y}\right), y\left(\frac{Q}{2}+y Q_{y}\right)\right)} .
$$

Note that for $\delta_{2}$ small enough,

$$
\frac{1}{2} \int\left(\varepsilon_{y}^{2}+\varepsilon^{2}\right) \leq \int \varepsilon_{1 y}^{2}+\varepsilon_{1}^{2} \leq 2 \int\left(\varepsilon_{y}^{2}+\varepsilon^{2}\right) .
$$

By bilinearity,

$$
\begin{aligned}
H_{\infty}(\varepsilon, \varepsilon)= & H_{\infty}\left(\varepsilon_{1}, \varepsilon_{1}\right)+H_{\infty}\left(\varepsilon_{2}, \varepsilon_{2}\right)+3 \int \varepsilon_{1 y} \varepsilon_{2 y} \\
& +\int \varepsilon_{1} \varepsilon_{2}-5 \int Q^{4} \varepsilon_{1} \varepsilon_{2}+20 \int y Q_{y} Q^{3} \varepsilon_{1} \varepsilon_{2} .
\end{aligned}
$$

We have from (54), (57), (56),

$$
\begin{aligned}
H_{\infty}\left(\varepsilon_{1}, \varepsilon_{1}\right) & \geq \delta_{1} \int\left(\varepsilon_{1 y}^{2}+\varepsilon_{1}^{2}\right) \geq \frac{\delta_{1}}{2} \int\left(\varepsilon_{y}^{2}+\varepsilon^{2}\right), \\
H_{\infty}\left(\varepsilon_{2}, \varepsilon_{2}\right) & =a^{2} H_{\infty}(Q, Q)+b^{2} H\left(y\left(\frac{Q}{2}+y Q_{y}\right), y\left(\frac{Q}{2}+y Q_{y}\right)\right) \\
& \leq C \delta_{2}^{2} \int\left(\varepsilon_{y}^{2}+\varepsilon^{2}\right) .
\end{aligned}
$$

Now, we have $\int \varepsilon_{1} \varepsilon_{2}=0$ and by integration by parts and (57),

$$
\left|3 \int \varepsilon_{1 y} \varepsilon_{2 y}-5 \int Q^{4} \varepsilon_{1} \varepsilon_{2}+20 \int y Q_{y} Q^{3} \varepsilon_{1} \varepsilon_{2}\right| \leq C(|a|+|b|)\left|\varepsilon_{1}\right|_{H^{1}} \leq C \delta_{2}|\varepsilon|_{H^{1}}^{2} .
$$

Thus for $\delta_{2}$ small, independent of $\varepsilon$, we have $H_{\infty}(\varepsilon, \varepsilon) \geq \frac{\delta_{1}}{4} \int\left(\varepsilon_{y}^{2}+\varepsilon^{2}\right)$. This proves the claim.

Step 2. Positivity of $H_{A}(\varepsilon, \varepsilon)$ for $A$ large. We claim: there exists $A_{0}>2$ such that for $A>A_{0}$, for all $\varepsilon \in H^{1}$ such that $(\varepsilon, Q)=\left(\varepsilon, y\left(\frac{Q}{2}+y Q_{y}\right)\right)=0$,

$$
H_{A}(\varepsilon, \varepsilon) \geq \frac{\delta_{1}}{8} \int\left(\varepsilon_{y}^{2} e^{-\frac{|y|}{A}}+\varepsilon^{2} e^{-\frac{|y|}{A}}\right) .
$$


The idea is to write $H_{A}(\varepsilon, \varepsilon)$ as $H_{\infty}\left(\varepsilon \sqrt{\Phi_{A}}, \varepsilon \sqrt{\Phi_{A}}\right)$ plus some error terms to be controlled. Then, to conclude, $\varepsilon \sqrt{\Phi_{A}}$ almost satisfies the orthogonality conditions, in the sense of step 1 .

Now,

$$
\int \varepsilon_{y}^{2} \Phi_{A}=\int\left(\varepsilon \sqrt{\Phi_{A}}\right)_{y}^{2}-\frac{1}{4} \int \varepsilon^{2} \frac{\Phi_{A}^{\prime 2}}{\Phi_{A}}-\int \varepsilon \varepsilon_{y} \Phi_{A}^{\prime},
$$

and

$$
\int Q_{y} Q^{3} \Psi_{A} \varepsilon^{2}=\int y Q_{y} Q^{3}\left(\varepsilon \sqrt{\Phi_{A}}\right)^{2}+\int Q_{y} Q^{3}\left(\Psi_{A}-y \Phi_{A}\right) \varepsilon^{2} .
$$

Therefore

$$
\begin{aligned}
H_{A}(\varepsilon, \varepsilon)= & H_{\infty}\left(\varepsilon \sqrt{\Phi_{A}}, \varepsilon \sqrt{\Phi_{A}}\right) \\
& -\frac{3}{8} \int \varepsilon^{2} \frac{\Phi_{A}^{\prime}{ }^{2}}{\Phi_{A}}-\frac{3}{2} \int \varepsilon \varepsilon_{y} \Phi_{A}^{\prime}+10 \int Q_{y} Q^{3}\left(\Psi_{A}-y \Phi_{A}\right) \varepsilon^{2} .
\end{aligned}
$$

Now we verify that $\varepsilon \sqrt{\Phi_{A}}$ satisfies (55) for $A$ large:

$$
\begin{aligned}
\left|\int Q \varepsilon \sqrt{\Phi_{A}}\right| & =\left|\int Q \varepsilon+\int Q\left(\sqrt{\Phi_{A}}-1\right) \varepsilon\right| \\
& =\left|\int Q\left(\sqrt{\Phi_{A}}-1\right) \varepsilon\right| \leq C e^{-\frac{A}{2}}\left|\varepsilon \sqrt{\Phi_{A}}\right|_{L^{2}},
\end{aligned}
$$

since $\Phi_{A}=1$ on $[-A, A], 0<\Phi_{A} \leq 1$ on $\mathbf{R}$, and the decay property of $Q$. Similarly, $\left|\int y\left(\frac{Q}{2}+y Q_{y}\right) \varepsilon \sqrt{\Phi_{A}}\right|=\left|\int y\left(\frac{Q}{2}+y Q_{y}\right)\left(\sqrt{\Phi_{A}}-1\right) \varepsilon\right| \leq C e^{-\frac{A}{4}}\left|\varepsilon \sqrt{\Phi_{A}}\right|_{L^{2}}$. Therefore, for $A$ large enough (depending only on $\delta_{1}$ ), $\varepsilon \sqrt{\Phi_{A}}$ satisfies the condition in (55). Thus

$$
\begin{aligned}
H_{\infty}\left(\varepsilon \sqrt{\Phi_{A}}, \varepsilon \sqrt{\Phi_{A}}\right) & \geq \frac{\delta_{1}}{4} \int\left(\varepsilon \sqrt{\Phi_{A}}\right)_{y}^{2}+\varepsilon^{2} \Phi_{A} \\
& =\frac{\delta_{1}}{4} \int\left(\varepsilon_{y}^{2} \Phi_{A}+\varepsilon^{2} \Phi_{A}\right)+\frac{\delta_{1}}{16} \int \varepsilon^{2} \frac{\Phi_{A}^{\prime}}{\Phi_{A}}+\frac{\delta_{1}}{4} \int \varepsilon \varepsilon_{y} \Phi_{A}^{\prime},
\end{aligned}
$$

and so

$$
\begin{aligned}
H_{A}(\varepsilon, \varepsilon) \geq & \frac{\delta_{1}}{4} \int\left(\varepsilon_{y}^{2} \Phi_{A}+\varepsilon^{2} \Phi_{A}\right)-\frac{3}{8} \int \varepsilon^{2} \frac{\Phi_{A}^{\prime 2}}{\Phi_{A}}+\left(\frac{\delta_{1}}{4}-\frac{3}{2}\right) \int \varepsilon \varepsilon_{y} \Phi_{A}^{\prime} \\
& -10\left|\int Q_{y} Q^{3}\left(\Psi_{A}-y \Phi_{A}\right) \varepsilon^{2}\right|
\end{aligned}
$$

Note that $0 \leq\left|\Phi_{A}^{\prime}\right| \leq \frac{C}{A} \Phi_{A}$, and next, since $\Psi_{A}-y \Phi_{A}=0$ on $[-A, A]$ and $\left|\Psi_{A}-y \Phi_{A}\right| \leq C A$, we have

$\left|\int Q_{y} Q^{3}\left(\Psi_{A}-y \Phi_{A}\right) \varepsilon^{2}\right| \leq C \sup \left(e^{-|x|}\left|\Psi_{A}-y \Phi_{A}\right|\right) \int Q^{3} \varepsilon^{2} \leq C A e^{-A} \int \varepsilon^{2} e^{-|y|}$.

Therefore, for $A$ large enough (depending on $\delta_{1}$ ),

$$
H_{A}(\varepsilon, \varepsilon) \geq \frac{\delta_{1}}{8} \int\left(\varepsilon_{y}^{2} \Phi_{A}+\varepsilon^{2} \Phi_{A}\right) \geq \frac{\delta_{1}}{8} \int\left(\varepsilon_{y}^{2} e^{-\frac{|y|}{A}}+\varepsilon^{2} e^{-\frac{|y|}{A}}\right) .
$$


Step 3. We remove the orthogonality condition $(\varepsilon, Q)=0$ using a similar argument to the one of step 1 . Let $\varepsilon$ be such that $\left(\varepsilon, y\left(\frac{Q}{2}+y Q_{y}\right)\right)=0$. Let $\varepsilon=\varepsilon_{1}+a Q$, where $a=(\varepsilon, Q) /(Q, Q)$. Then

$$
\begin{aligned}
H_{A}(\varepsilon, \varepsilon)= & H_{A}\left(\varepsilon_{1}, \varepsilon_{1}\right)+a^{2} H_{A}(Q, Q)+3 a \int Q_{y} \Phi_{A} \varepsilon_{1 y}+a \int Q \Phi_{A} \varepsilon_{1} \\
& -5 a \int Q^{5} \Phi_{A} \varepsilon_{1}+20 a \int Q_{y} Q^{4} \Psi_{A} \varepsilon_{1} \\
\geq & \frac{\delta_{1}}{8} \int\left(\varepsilon_{1 y}^{2} e^{-\frac{|y|}{A}}+\varepsilon_{1}^{2} e^{-\frac{|y|}{A}}\right)-C a^{2}-C|a| \int\left(\left|\varepsilon_{1}\right|+\left|\varepsilon_{1 y}\right|\right) e^{-|y|} e^{-\frac{|y|}{2 A}} \\
\geq & \frac{\delta_{1}}{8} \int\left(\varepsilon_{1 y}^{2} e^{-\frac{|y|}{A}}+\varepsilon_{1}^{2} e^{-\frac{|y|}{A}}\right)-C a^{2}-C|a|\left(\int\left(\varepsilon_{1 y}^{2} e^{-\frac{|y|}{A}}+\varepsilon_{1}^{2} e^{-\frac{|y|}{A}}\right)\right)^{1 / 2} \\
\geq & \frac{\delta_{1}}{16} \int\left(\varepsilon_{1 y}^{2} e^{-\frac{|y|}{A}}+\varepsilon_{1}^{2} e^{-\frac{|y|}{A}}\right)-C a^{2} \\
\geq & \frac{\delta_{1}}{32} \int\left(\varepsilon_{y}^{2} e^{-\frac{|y|}{A}}+\varepsilon^{2} e^{-\frac{|y|}{A}}\right)-C a^{2}-C|a|\left(\int\left(\varepsilon_{y}^{2} e^{-\frac{|y|}{2 A}}+\varepsilon^{2} e^{-\frac{|y|}{2 A}}\right)\right)^{1 / 2} \\
\geq & \frac{\delta_{1}}{64} \int\left(\varepsilon_{y}^{2} e^{-\frac{|y|}{A}}+\varepsilon^{2} e^{-\frac{|y|}{A}}\right)-C(\varepsilon, Q)^{2} .
\end{aligned}
$$

Step 4. Control of the remainding terms. Now, we are reduced to showing that by possibly taking a larger $A$ and a smaller $\alpha_{0}$, we can control the perturbation terms in the expression of $\left(\int \Psi_{A} \varepsilon^{2}\right)_{s}$ by an arbitrarily small constant multiplied by $\int\left(\varepsilon_{y}^{2} e^{-\frac{|y|}{A}}+\varepsilon^{2} e^{-\frac{|y|}{A}}\right)$.

First, $\left|\Phi_{A}^{\prime \prime}(y)\right|=\frac{1}{A^{2}}\left|\Phi^{\prime \prime}\left(\frac{x}{A}\right)\right| \leq \frac{C}{A^{2}} \Phi_{A}(y)$, so that $\left|\int \varepsilon^{2} \Phi_{A}^{\prime \prime}\right| \leq \frac{C}{A^{2}} \int \varepsilon^{2} \Phi_{A}$.

Second, we have by Lemma 2

and

$$
\left|\frac{\lambda_{s}}{\lambda}\right|+\left|\frac{x_{s}}{\lambda}-1\right| \leq C\left(\int \varepsilon^{2} e^{-\frac{|y|}{2}}\right)^{1 / 2}
$$

$$
\begin{aligned}
\left|\int\left(\frac{Q}{2}+y Q_{y}\right) \Psi_{A} \varepsilon\right| & =\left|\int\left(\frac{Q}{2}+y Q_{y}\right)\left(\Psi_{A}-y\right) \varepsilon\right| \\
& \leq \sup \left(\left|\Psi_{A}-y\right| e^{-\frac{|y|}{4}}\right) \int e^{-\frac{|y|}{4}|\varepsilon|} \\
& \leq C e^{-\frac{A}{8}}\left(\int \varepsilon^{2} e^{-\frac{|y|}{2}}\right)^{1 / 2} .
\end{aligned}
$$

Similarly $\left|\int Q_{y} \Psi_{A} \varepsilon\right| \leq C e^{-\frac{A}{8}}\left(\int \varepsilon^{2} e^{-\frac{|y|}{2}}\right)^{1 / 2}$.

Next, since from Lemma $2,|\varepsilon|_{L^{\infty}} \leq|\varepsilon|_{H^{1}}^{1 / 2}|\varepsilon|_{L^{2}}^{1 / 2} \leq C \sqrt{\alpha_{0}}$, we have

$$
\left|K_{A}(\varepsilon)\right| \leq C \sqrt{\alpha_{0}} \int \varepsilon^{2} \Phi_{A}, \quad \text { and } \quad \int \Phi_{A} \varepsilon^{6} \leq \alpha_{0}^{2} \int \varepsilon^{2} \Phi_{A} .
$$


Finally, since $|y| e^{-\frac{|y|}{A}} \leq C A e^{-\frac{|y|}{2 A}}$,

$$
\begin{aligned}
\left|\frac{\lambda_{s}}{\lambda} \int y \Phi_{A} \varepsilon^{2}\right| & \leq C\left(\int e^{-\frac{|y|}{2}} \varepsilon^{2}\right)^{1 / 2} A \int e^{-\frac{|y|}{2 A}} \varepsilon^{2} \\
& \leq C A\left(\int \varepsilon^{2}\right)^{1 / 2} \int \varepsilon^{2} e^{-\frac{|y|}{A}} \leq C \sqrt{\alpha_{0}} A \int \varepsilon^{2} e^{-\frac{|y|}{A}} .
\end{aligned}
$$

Therefore, we can fix $A_{0}>2$ large (independent of $\alpha_{0}$ ) so that

$$
\frac{1}{2}\left(\int \Psi_{A_{0}} \varepsilon^{2}\right)_{s} \leq-\frac{\delta_{1}}{128} \int\left(\varepsilon_{y}^{2} e^{-\frac{|y|}{A_{0}}}+\varepsilon^{2} e^{-\frac{|y|}{A_{0}}}\right)+C^{\prime \prime}(\varepsilon, Q)^{2}+\sqrt{\alpha_{0}} A_{0} \int \varepsilon^{2} e^{-\frac{|y|}{A_{0}}} .
$$

The constant $A_{0}>2$ being fixed, we take $\alpha_{0}>0$ small enough so that

$$
\frac{1}{2}\left(\int \Psi_{A_{0}} \varepsilon^{2}\right)_{s} \leq-\frac{\delta_{1}}{256} \int\left(\varepsilon_{y}^{2} e^{-\frac{|y|}{A_{0}}}+\varepsilon^{2} e^{-\frac{|y|}{A_{0}}}\right)+C^{\prime \prime}(\varepsilon, Q)^{2} .
$$

Thus the proposition is proved.

\section{Appendix B}

Proof of Lemma 9. Note first that for all $x_{1}<0, n \geq 0, t \in\left[0, T_{n}\right)$, $0 \leq \mathcal{J}_{x_{0}, n}(t) \leq 2 \int Q^{2}$.

Step 1. We show that for $n \geq n_{0}$ fixed, the function $\mathcal{J}_{x_{0}, n}(t)$ has a limit $\mathcal{J}_{x_{0}, n}$ as $t \uparrow T_{n}$. Since $\lambda_{n}(t) \leq 1$, by the proof of Lemma 5 applied to $u_{n}$,

for all $x_{1}<-a_{1}$, for all $t \in\left[0, T_{n}\right), \quad \mathcal{J}_{x_{1}, n}^{\prime}(t) \geq-C x_{n t} e^{-\frac{\left|x_{1}\right|}{3}} e^{-\frac{1}{4} x_{n}(t)}$. Now, for all $x_{1}<-a_{1}$, for all $t^{\prime}, t \in\left[0, T_{n}\right), t^{\prime}<t$,

$$
\mathcal{J}_{x_{1}, n}(t)-\mathcal{J}_{x_{1}, n}\left(t^{\prime}\right) \geq C e^{-\frac{\left|x_{1}\right|}{3}}\left(e^{-\frac{1}{4} x_{n}(t)}-e^{-\frac{1}{4} x_{n}\left(t^{\prime}\right)}\right) .
$$

Therefore the function $t \mapsto \mathcal{J}_{x_{1}, n}(t)-C e^{-\frac{\left|x_{1}\right|}{3}} e^{-\frac{1}{4} x_{n}(t)}$ is nondecreasing and bounded on $\left[0, T_{n}\right)$. Thus it has a limit as $t \uparrow T_{n}$. Since $e^{-\frac{1}{4} x_{n}(t)}$ has a limit as $t \uparrow T_{n}\left(x_{n t} \geq 0\right)$, this concludes the proof.

Step 2. We claim that there exists $C>0$ such that for all $x_{1}, x_{2}<0$, $n_{2}>n_{0}, \delta_{1}>0$, there exists $n_{1}=n_{1}\left(x_{1}, x_{2}, n_{2}, \delta_{1}\right)$ such that

$$
\text { for all } t \in\left[0, T_{n}\right) \text {, for all } n \geq n_{1}, \quad \mathcal{J}_{x_{1}, n}(t) \geq \mathcal{J}_{x_{2}, n_{2}}-\delta_{1}-C e^{\frac{x_{2}}{8}} \text {. }
$$

Proof of claim (59). We use the recurrence of $u_{n}(t)$. Let $x_{1}, x_{2}, n_{2}$ and $\delta_{1}$ be as in the claim. Let $n \geq n_{1} \geq n_{2}$, with $n_{1}$ to be chosen later. We have by direct calculations

$$
u_{n}(t, x)=\lambda^{1 / 2}\left(t_{n}\right) u\left(t_{n}+\lambda^{3}\left(t_{n}\right) t, \lambda\left(t_{n}\right) x+x\left(t_{n}\right)\right),
$$


and

$$
u(t, x)=\frac{1}{\lambda^{1 / 2}\left(t_{n_{2}}\right)} u_{n_{2}}\left(\frac{t-t_{n_{2}}}{\lambda^{3}\left(t_{n_{2}}\right)}, \frac{x-x\left(t_{n_{2}}\right)}{\lambda\left(t_{n_{2}}\right)}\right),
$$

so that

$$
u_{n}(t, x)=\frac{\lambda^{1 / 2}\left(t_{n}\right)}{\lambda^{1 / 2}\left(t_{n_{2}}\right)} u_{n_{2}}\left(\frac{\lambda^{3}\left(t_{n}\right)}{\lambda^{3}\left(t_{n_{2}}\right)} t+\frac{t_{n}-t_{n_{2}}}{\lambda^{3}\left(t_{n_{2}}\right)}, \frac{\lambda\left(t_{n}\right)}{\lambda\left(t_{n_{2}}\right)} x+\frac{x\left(t_{n}\right)-x\left(t_{n_{2}}\right)}{\lambda\left(t_{n_{2}}\right)}\right) .
$$

Note that

$$
\lambda_{n}(t)=\frac{\lambda\left(t_{n}+\lambda^{3}\left(t_{n}\right) t\right)}{\lambda\left(t_{n}\right)}, \quad x_{n}(t)=\frac{x\left(t_{n}+\lambda^{3}\left(t_{n}\right) t\right)-x\left(t_{n}\right)}{\lambda\left(t_{n}\right)} .
$$

By the change of variable $y=\frac{\lambda\left(t_{n}\right)}{\lambda\left(t_{n_{2}}\right)} x+\frac{x\left(t_{n}\right)-x\left(t_{n_{2}}\right)}{\lambda\left(t_{n_{2}}\right)}$, we obtain

$$
\begin{aligned}
\mathcal{J}_{x_{1}, n}(t) & =\int u_{n}^{2}(t, x)\left(1-\psi\left(x-x_{n}\left(t_{0}\right)-x_{1}-\frac{1}{4}\left(x_{n}(t)-x_{n}\left(t_{0}\right)\right)\right)\right) d x \\
& =\int u_{n_{2}}^{2}\left(\hat{t}_{n}, y\right)\left(1-\psi\left(\frac{\lambda\left(t_{n_{2}}\right)}{\lambda\left(t_{n}\right)}\left(y-y_{1 n}\right)\right)\right) d y
\end{aligned}
$$

where $\hat{t}_{n}=\frac{\lambda^{3}\left(t_{n}\right)}{\lambda^{3}\left(t_{n_{2}}\right)} t+\frac{t_{n}-t_{n_{2}}}{\lambda^{3}\left(t_{n_{2}}\right)}$, and $y_{1 n}=\frac{x\left(t_{n}\right)-x\left(t_{n_{2}}\right)}{\lambda\left(t_{n_{2}}\right)}+\frac{\lambda\left(t_{n}\right)}{\lambda\left(t_{n_{2}}\right)} x_{n}\left(t_{0}\right)+$ $\frac{\lambda\left(t_{n}\right)}{\lambda\left(t_{n_{2}}\right)} x_{1}+\frac{1}{4} \frac{\lambda\left(t_{n}\right)}{\lambda\left(t_{n_{2}}\right)}\left(x_{n}(t)-x_{n}\left(t_{0}\right)\right)$.

In view of the preceding calculations, it is natural to compare $\mathcal{J}_{x_{1}, n}(t)$ to $\mathcal{J}_{x_{2}, n_{2}}\left(\hat{t}_{n}\right)$. Note that

$$
\mathcal{J}_{x_{2}, n_{2}}\left(\hat{t}_{n}\right)=\int u_{n_{2}}^{2}\left(\hat{t}_{n}, y\right)\left(1-\psi\left(y-y_{2 n}\right)\right) d y
$$

where $y_{2 n}=x_{n_{2}}\left(t_{0}\right)+x_{2}+\frac{1}{4}\left(x_{n_{2}}\left(\hat{t}_{n}\right)-x_{n_{2}}\left(t_{0}\right)\right)$. Now,

$$
\begin{aligned}
y_{1 n} & =-\frac{x\left(t_{n_{2}}\right)}{\lambda\left(t_{n_{2}}\right)}+\frac{3}{4} \frac{x\left(t_{n}+\lambda^{3}\left(t_{n}\right) t_{0}\right)}{\lambda\left(t_{n_{2}}\right)}+\frac{\lambda\left(t_{n}\right)}{\lambda\left(t_{n_{2}}\right)} x_{1}+\frac{1}{4} \frac{x\left(t_{n}+\lambda^{3}\left(t_{n}\right) t\right)}{\lambda\left(t_{n_{2}}\right)}, \\
y_{2 n} & =\frac{3}{4} \frac{x\left(t_{n_{2}}+\lambda^{3}\left(t_{n_{2}}\right) t_{0}\right)}{\lambda\left(t_{n_{2}}\right)}-\frac{x\left(t_{n_{2}}\right)}{\lambda\left(t_{n_{2}}\right)}+x_{2}+\frac{1}{4} \frac{x\left(t_{n}+\lambda^{3}\left(t_{n}\right) t\right)}{\lambda\left(t_{n_{2}}\right)}, \\
y_{1 n}-y_{2 n} & =\frac{3}{4} \frac{x\left(t_{n}+\lambda^{3}\left(t_{n}\right) t_{0}\right)-x\left(t_{n_{2}}+\lambda^{3}\left(t_{n_{2}}\right) t_{0}\right)}{\lambda\left(t_{n_{2}}\right)}+\frac{\lambda\left(t_{n}\right)}{\lambda\left(t_{n_{2}}\right)} x_{1}-x_{2} .
\end{aligned}
$$

Note that $t_{n}+\lambda^{3}\left(t_{n}\right) t_{0} \rightarrow T$ as $n \rightarrow+\infty$. Therefore, there exists $n_{0}=n_{0}\left(n_{2}\right)$ such that $x\left(t_{n}+\lambda^{3}\left(t_{n}\right) t_{0}\right)-x\left(t_{n_{2}}+\lambda^{3}\left(t_{n_{2}}\right) t_{0}\right) \geq 0$. For $n_{1}$ large depending on $x_{1}, x_{2}, n_{2}$, we also have $\frac{\lambda\left(t_{n}\right)}{\lambda\left(t_{n_{2}}\right)} x_{1} \geq x_{2} / 2$. Thus

$$
y_{1 n}-y_{2 n} \geq-\frac{x_{2}}{2} .
$$


Since $\psi^{\prime} \geq 0, x_{2}<0, \frac{\lambda\left(t_{n_{2}}\right)}{\lambda\left(t_{n}\right)}>1$, and $1-\psi\left(y-y_{2 n}\right) \leq C e^{\frac{x_{2}}{4 \sqrt{3}}} \leq C e^{\frac{x_{2}}{8}}$, for $y>y_{2 n}-\frac{x_{2}}{2}$, we obtain

$$
\begin{aligned}
\mathcal{J}_{x_{1}, n}(t) & \geq \int u_{n_{2}}^{2}\left(\hat{t}_{n}, y\right)\left(1-\psi\left(\frac{\lambda\left(t_{n_{2}}\right)}{\lambda\left(t_{n}\right)}\left(y-y_{2 n}+\frac{x_{2}}{2}\right)\right)\right) d y \\
& \geq \int_{y<y_{2 n}-\frac{x_{2}}{2}} u_{n_{2}}^{2}\left(\hat{t}_{n}, y\right)\left(1-\psi\left(y-y_{2 n}\right)\right) d y \\
& \geq \int u_{n_{2}}^{2}\left(\hat{t}_{n}, y\right)\left(1-\psi\left(y-y_{2 n}\right)\right) d y-C e^{\frac{x_{2}}{8}}
\end{aligned}
$$

Therefore,

$$
\mathcal{J}_{x_{1}, n}(t) \geq \mathcal{J}_{x_{2}, n_{2}}\left(\hat{t}_{n}\right)-C e^{\frac{x_{2}}{8}},
$$

where $C$ is a constant. Now, since $\hat{t}_{n} \rightarrow \frac{T-t_{n_{2}}}{\lambda^{3}\left(t_{n_{2}}\right)}=T_{n_{2}}$, it is sufficient to consider a possibly larger $n_{1}$ (depending on $\delta_{1}$ ) such that $\mathcal{J}_{x_{2}, n_{2}}\left(\hat{t}_{n}\right) \geq \mathcal{J}_{x_{2}, n_{2}}-\delta_{1}$. Thus claim (59) is proved.

Step 3. Conclusion of the proof. We use the claim, with $x_{1}, x_{2}<0$. Passing to the limit $t \rightarrow T_{n}$, we see that

$$
\mathcal{J}_{x_{1}, n} \geq \mathcal{J}_{x_{2}, n_{2}}-\delta_{1}-C e^{\frac{x_{2}}{8}} .
$$

Then $\underline{\lim }_{n \rightarrow+\infty} \mathcal{J}_{x_{1}, n} \geq \mathcal{J}_{x_{2}, n_{2}}-\delta_{1}-C e^{\frac{x_{1}}{8}}$, and next, $\underline{\lim }_{n \rightarrow+\infty} \mathcal{J}_{x_{1}, n} \geq$ $\varlimsup_{n \rightarrow+\infty} \mathcal{J}_{x_{2}, n}-\delta_{1}-C e^{\frac{x_{1}}{8}}$. Finally, by $\delta_{1} \rightarrow 0$, we obtain

$$
\underline{\lim }_{n \rightarrow+\infty} \mathcal{J}_{x_{1}, n} \geq \varlimsup_{n \rightarrow+\infty} \mathcal{J}_{x_{2}, n}-C e^{\frac{x_{1}}{8}} .
$$

Note that since for all $t, \mathcal{J}_{x_{1}, n}(t)$ is nondecreasing in $x_{1}$, the limit $\mathcal{J}_{x_{1}, n}$ is also nondecreasing in $x_{1}$, and $\varlimsup_{n \rightarrow+\infty} \mathcal{J}_{x_{1}, n}$ is still nondecreasing in $x_{1}$. We consider

$$
\mathcal{J}=\lim _{x_{1} \rightarrow-\infty}\left(\varlimsup_{n \rightarrow+\infty} \mathcal{J}_{x_{1}, n}\right) .
$$

Note that by $(60)$, we also have $\mathcal{J}=\lim _{x_{1} \rightarrow-\infty}\left(\underline{\lim }_{n \rightarrow+\infty} \mathcal{J}_{x_{1}, n}\right)$.

First, we prove the lower estimate in (49), using step 2 and the definition of $\mathcal{J}$. By $\varliminf_{n \rightarrow+\infty} \mathcal{J}_{x_{1}, n} \geq \mathcal{J}$, and (59), for $n_{2}$ large enough depending on $\delta_{1}$, we have for all $n \geq n_{1}\left(n_{2}, x_{1}\right)$, for all $t \in\left[0, T_{n}\right)$,

$$
\begin{aligned}
\mathcal{J}_{x_{1}, n}(t) & \geq \mathcal{J}_{x_{1}, n_{2}}-\delta_{1}-C e^{\frac{x_{1}}{8}} \\
& \geq \underline{\lim }_{n \rightarrow+\infty} \mathcal{J}_{x_{1}, n}-2 \delta_{1}-C e^{\frac{x_{1}}{8}} \geq \mathcal{J}-2 \delta_{1}-C e^{\frac{x_{1}}{8}} .
\end{aligned}
$$

Finally, we prove the upper estimate in (49), by using Lemma 5. Indeed, by this lemma, we have for all $t, t^{\prime} \in\left[0, T_{n}\right), t<t^{\prime}, \quad \mathcal{J}_{x_{1}, n}(t) \leq \mathcal{J}_{x_{1}, n}\left(t^{\prime}\right)+$ $C e^{\frac{x_{1}}{3}}$.

Since $t^{\prime} \rightarrow T_{n}$, we obtain for all $t \in\left[0, T_{n}\right), \quad \mathcal{J}_{x_{1}, n}(t) \leq \mathcal{J}_{x_{1}, n}+C e^{\frac{x_{1}}{3}}$. Therefore, for $n$ large depending on $\delta_{1}$ : for all $t \in\left[0, T_{n}\right), \mathcal{J}_{x_{1}, n}(t) \leq$ $\varlimsup_{n \rightarrow+\infty} \mathcal{J}_{x_{1}, n}+\delta_{1}+C e^{\frac{x_{1}}{3}}$. 
Note that by (60), we have $\overline{\lim }_{n \rightarrow+\infty} \mathcal{J}_{x_{1}, n} \leq \underline{\lim }_{n \rightarrow+\infty} \mathcal{J}_{x_{2}, n}+C e^{\frac{x_{1}}{8}}$, and so, by $x_{2} \rightarrow+\infty: \varlimsup_{n \rightarrow+\infty} \mathcal{J}_{x_{1}, n} \leq \mathcal{J}+C e^{\frac{x_{1}}{8}}$. Therefore,

$$
\text { for all } t \in\left[0, T_{n}\right), \quad \mathcal{J}_{x_{1}, n}(t) \leq \mathcal{J}+C e^{\frac{x_{1}}{8}}+\delta_{1}+C e^{\frac{x_{1}}{3}} .
$$

This concludes the proof of the lemma.

Université de Cergy-Pontoise, Cergy-Pontoise, France

E-mail address: Yvan.Martel@math.u-cergy.fr

Université de Cergy-Pontoise, Cergy-Pontoise, France

Institut Universitaire de France, Paris, France

E-mail address: Frank.Merle@math.u-cergy.fr

\section{REFERENCES}

[1] J. L. Bona, V. A. Dougalis, O. A. Karakashian, and W. R. McKinney, Conservative, high order numerical schemes, Philos. Trans. Roy. Soc. London Ser. A 351 (1995), 107-164.

[2] J. L Bona and F. B. Weissler, Similarity solutions of the generalized Korteweg-de Vries equations, Math. Proc. Cambridge Philos. Soc. 127 (1999), 323-351.

[3] J. Bourgain, Harmonic analysis and nonlinear partial differential equations, Proc. Internat. Congress of Mathematicians 1, 2 (Zürich, 1994), 31-44, Birkhäuser, Basel, 1995.

[4] C. Fermanian-Kammerer, F. Merle, and H. ZaAg, Stability of the blow-up profile of nonlinear heat equations from the dynamical system point of view, Math. Ann. 317 (2000), $347-387$.

[5] Y. Giga and R. Konn, Nondegeneracy of blowup for semilinear heat equations, Comm. Pure Appl. Math. 42 (1989), 845-884.

[6] J. Ginibre and Y. Tsutsumi, Uniqueness of solutions for the generalized Kortewegde Vries equation, SIAM J. Math. Anal. 20 (1989), 1388-1425.

[7] T. Kato, On the Cauchy problem for the (generalized) Korteweg-de Vries equation, Adv. in Math. Suppl. Studies, Studies in Applied Math. 8 (1983), 93-128.

[8] C. E. Kenig, G. Ponce, and L. Vega, Well-posedness and scattering results for the generalized Korteweg-de Vries equation via the contraction principle, Comm. Pure Appl. Math. 46 (1993), 527-620.

[9] D. J. Korteweg and G. De Vries, On the change of form of long waves advancing in a rectangular canal, and on a new type of long stationary waves, Philos. Mag. 539 (1895), 422-443.

[10] P. D. LAx, Integrals of nonlinear equations of evolution and solitary waves, Comm. Pure Appl. Math. 21 (1968), 467-490.

[11] Y. Martel and F. Merle, Instability of solitons for the critical generalized Kortewegde Vries equation, Geom. Funct. Anal. 11 (2001), 74-123.

[12] A Liouville theorem for the critical generalized Korteweg-de Vries equation, J. Math. Pures Appl. 79 (2000), 339-425.

[13] F. Merle, Lower bounds for the blowup rate of solutions of the Zakharov equation in dimension two, Comm. Pure Appl. Math. 49 (1996), 765-794.

[14] Blow-up phenomena for critical nonlinear Schrödinger and Zakharov equations, Proc. Internat. Congress of Mathematicians (Berlin, 1998), Doc. Math. 1998, 57-66.

[15] Existence of blow-up solutions in the energy space for the critical generalized KdV equation, J. Amer. Math. Soc. 14 (2001), 555-578.

[16] R. M. Miura, The Korteweg-de Vries equation: a survey of results, SIAM Review 18 (1976), 412-459. 
[17] M. I. Weinstein, Nonlinear Schrödinger equations and sharp interpolation estimates, Comm. Math. Phys. 87 (1983), 567-576.

(Received October 16, 2000) 